\title{
Escape from a Metastable State
}

\author{
Peter Hanggi ${ }^{1}$
}

Received July 11, 1985

\begin{abstract}
Many important processes in science involve the escape of a particle over a barrier. In this review, we report, extend, and interpret various theories of noiseactivated escape. We discuss the connection between many-body transition state theory and Kramers' original diffusive Brownian motion approach (both in oneand multidimensional potential fields) and emphasize the physical situation inherent in Kramers' rate for weak friction. A rate theory accounting for memory friction is presented together with a set of criteria which test its validity. The complications and peculiarities of noise-activated escape in driven systems exhibiting multiple, locally stable stationary nonequilibrium states are identified and illustrated. At lower temperatures, quantum tunneling effects begin to play an increasingly important role. Early approaches and more recent developments of the quantum version of Kramers approach are discussed, thereby providing a description for dissipative escape at all temperatures.
\end{abstract}

KEY WORDS: Many-body rate theory; Kramers rate; bistability; multidimensional metastability; driven systems; quantum tunneling; imaginary time path integrals.

\section{INTRODUCTION}

The problem of escape from a metastable state plays a central role in many scientific areas, most notably in chemical kinetics, the theory of diffusion in solids, transport in biomolecules, electron transport in semiconductors, and the dynamics in nonlinear optics to name only a few. The problem not only arises in a countless number of systems modeling escape from a metastable equilibrium state driven by thermal fluctuations, but also in driven systems characterized by nonthermal steady states which may exhibit competing states of local stability.

\footnotetext{
${ }^{1}$ Department of Physics, Polytechnic Institute of New York, 333 Jay Street, Brooklyn, New York 11201.
} 
The common theme underlying the description of escape processes is based on Brownian motion. In adapting this scheme, the motion of the principal degrees of freedom is treated explicitly while the interaction with other degrees of freedom, including those of the heat bath(s) coupled to the system of interest, are represented by frictional forces and noise; i.e., the deterministic equations governing the dynamics of the escape process must be complemented by random forces. These random forces present the key input allowing the system to get away from preferred states of local stability. Generally, these random forces are rather weak; i.e., the particle trajectories are localized for most of the time around the states of local stability before there occurs an occasional, fluctuation-induced excursion over a barrier toward a neighboring state of local stability. Owing to such a clear-cut time separation, the escape dynamics can in most cases be adequately described by a single rate coefficient. This viewpoint is principally due to Kramers. ${ }^{(1)}$ Over a long period, Kramers' work was only appreciated by a few theoreticians. ${ }^{(2-5)}$ Recently, however, Kramers' ideas have resurfaced in many theoretical ${ }^{(6,7) 2,3}$ and experimental studies ${ }^{(8-21)}$ of kinetics in a variety of physical systems.

In the following sections I have attempted to review the present status of the field as well as to survey some recent, interesting developments. In this paper I generally focus on an escape dynamics which is governed by a low-dimensional reaction path in the state space of the relevant degrees of freedom and assume only a finite number of competing states of local stability, separated from each other by (multidimensional) saddle point configurations.

\section{CLASSICAL TREATMENT}

\subsection{Transition State and Many-Body Rate Theory}

Neglecting quantum effects, the classical treatment is based on an analysis of the basic process whereby a particle jumps from one locally stable position to a neighboring locally stable position. The traditional description of this escape process is termed transition state theory (TST). ${ }^{(22-29)}$ This theory gives the probability of a particle jumping from one well to another by passing over a barrier as a product of two factors:

${ }^{2}$ In the last $10-15$ years, the theoretical papers concerned with Kramers' approach has become a flood. Some articles which provide additional interesting insight into the conventional Kramers' approach are listed in Ref. 6.

${ }^{3}$ For the recent, interesting theoretical extension of Kramers' work to non-Markovian situations (memory damping) see the references given in Section 2.6, e.g., Refs. 56, 61, 65-70 and 72; for driven systems see Refs. 91 and 92. 
The Arrhenius factor $B$, and a prefactor $A$. The Arrhenius factor ${ }^{4}$

$$
B=\exp \left(-E_{b} / k T\right)
$$

where $E_{b}$ is the barrier height, gives the probability of finding the escaping particle with energy higher than that of the barrier. The prefactor gives the probability per unit time of finding the particle "knocking" at the door before passing to the neighboring well. In a one-dimensional well, simple TST identifies the prefactor $A$ with the frequency of oscillation at the bottom of the well ${ }^{(22-25)}$.

$$
A=v_{0}=\frac{\omega_{0}}{2 \pi}
$$

Here, $v_{0}$ emerges from the equilibrium population inside the initial well. Thus, the common argument according to which $v_{0}$ is the frequency at which the particle tries to escape is somewhat meaningless. Note also that within simple TST the prefactor $A$ does not exhibit a dependence on transport coefficients such as a viscosity, etc. All of the later classical theoretical studies focus on a more detailed evaluation of this prefactor $A$.

A many-body generalization of the traditional formulation of transition state theory has been known for some time. ${ }^{(25-29)}$ Unfortunately, the advocates of many-body transition state theory ${ }^{(25 \cdot 28)}$ seem to have been unaware of the full content of Kramers' original work. Moreover, the broad applicability of many-body rate theory is limited in practice because of the substantial difficulties encountered in evaluating its parameters for practical applications. ${ }^{5}$ The key assumptions of many-body rate theory are of two kinds ${ }^{(29)}$ :

(1) Thermodynamic equilibrium prevails throughout the entire system of all degrees of freedom. Any effects of nonequilibrium which result in perturbations of the Boltzmann distribution are neglected.

(2) Correlated returns ("recrossings") of a system that has crossed the (parabolic) bounding hypersurface through a saddle point are neglected.

Taken together, these assumptions reduce the calculation of a rate coefficient, $\Gamma$, to a consideration of the equilibrium concentration of

\footnotetext{
${ }^{4}$ Plotting the logarithm of rate data for $\mathrm{CH}_{3} \mathrm{~J}+\mathrm{C}_{2} \mathrm{H}_{5} \mathrm{ONa} \rightarrow \mathrm{CH}_{3} \mathrm{OC}_{2} \mathrm{H}_{5}+\mathrm{NaJ}$, by Hecht and Conrad [W. Hecht and M. Conrad, Z. Phys. Chem. 3:450 (1889)], versus inverse temperature, Arrhenius [S. Arrhenius, Z. Phys. Chem. 4:226 (1889)], found a straight line (Arrhenius plot); i.e., $\Gamma=A \exp \left(-E_{b} / k T\right)$.

${ }^{5}$ In fairness, Kramers' theory uses a friction coefficient, which in many situations is not known a priori. However, the friction often can either be measured experimentally (e.g., electrical resistance) or evaluated approximately from first principles.
} 
activated configurations on an adiabatic potential surface. Using the assumptions (1) and (2), the rate $\Gamma$ takes on the form ${ }^{(25-29)}$

$$
\Gamma=v^{*} \exp \left(-E_{b} / k T\right)
$$

where $v^{*}$ is an effective frequency defined by

$$
v^{*}=\frac{\prod_{j=1}^{N} v_{j}^{0}}{\prod_{j=1}^{N-1} \bar{v}_{j}}
$$

Here, $N$ denotes the number of degrees of freedom of the system, the set $\left\{v_{j}^{0}\right\}$ are the characteristic frequencies of normal modes derived from a dynamical matrix relative to the parabolic minimum point of the potential energy. The set $\left\{\bar{v}_{j}\right\}$ are the characteristic frequencies of the stable, $(N-1)$ normal modes of the system which are constrained to lie in the (properly chosen) separating hypersurface through the saddle point. Within a full phase-space description, it is reasonable to assume the validity of assumption (2) if the considered temperatures are not too high. A difficulty of more fundamental nature results from the assumption of thermodynamic equilibrium throughout the escape process. In particular, it should be noted that the initial state is a peculiar deviation from equilibrium in the sense that the particle is localized in a well. As time evolves, we are interested in the evolution toward a "new state of equilibrium in a neighboring well" where the particle again is constrained. This evolution from one "metastable equilibrium" in the initial well to another, neighboring metastable equilibrium sets the time scale for the problem. A priori, it would be far fetched to assume that equilibrium prevails at all times throughout this process. In order for it to be true, we must assume that vertical thermalization (inside the well) is much more rapid than the horizontal outflow noted above. This point has been fully appreciated by Kramers $^{(1)}$; although somewhat less so by more recent authors. ${ }^{(28,30,31)}$

Owing to its intractability, present already in idealized model systems, ${ }^{(31)}$ the use of many-body rate theory [subject to the assumptions stated in (1) and (2) above] is likely not the most appropriate vehicle for thinking about escape in real situations. Of a more practical nature is an approach which uses a reduced description in terms of a few relevant coordinates which make up the so-called "reaction coordinate." Such a procedure entails new concepts which loosely can be characterized as friction and entropy. The entropy factor is related to the reduction of the highdimensional potential energy surface to a lower-dimensional potential energy surface (see Section 2.5 below). Friction describes a dynamical effect which incorporates the effects of the residual degrees of freedom lost upon contraction of the full phase space dynamics $\mathbf{\Omega}(t)$. Clearly, the two effects are not independent of each other. 
The mathematics of this reduction has been worked out in full generality within classical statistical mechanics. ${ }^{(32)}$ The reduced dynamics can be represented either in terms of a generalized master equation or in the form of a generalized Langevin equation. For a detailed account on this reduction and a discussion of the various interrelationships between the two approaches we refer the reader to Ref. 32. One of the results is that, depending on the form of the coupling between the escaping particle and the environmental degrees of freedom, there occurs a renormalization of mass as well as a renormalization of bare potential fields $-\ldots$ both are classical effects which are also of importance in a quantum treatment of the escape problem (see Section 3 ). This reduction of $\sim 10^{23}$ degrees of freedom contained in $\boldsymbol{\Omega}(t)$ onto a small set of relevant macrovariables $\mathbf{x}(t)$, the reaction coordinates $\mathbf{x}(t)$, yields the problem much more tractable. The exact equation of motion for $\mathbf{x}(t)$ takes the form ${ }^{(32,33)}$

$\dot{\mathbf{x}}_{t}=\mathbf{v}\left(\mathbf{x}_{t}\right)+k T \int_{0}^{t} \bar{p}^{-1}\left(\mathbf{x}_{t-s}\right) \frac{\partial}{\partial \mathbf{x}_{t-s}}\left[\zeta\left(\mathbf{x}_{t-s} ; s\right) \bar{p}\left(\mathbf{x}_{t-s}\right)\right] d s+\xi(t)$

The effect of mass renormalization is contained in a generalized, timedependent friction tensor $\zeta(\mathbf{x} ; s)$ (memory friction), while the renormalization of the bare potential occurs via a drift field

$$
\mathbf{v}(\mathbf{x})=\int \delta(\mathbf{x}(\mathbf{\Omega})-\mathbf{x}) \dot{\mathbf{x}}(\boldsymbol{\Omega})\left(\frac{\bar{p}(\boldsymbol{\Omega})}{\bar{p}(\mathbf{x})}\right) d \Omega
$$

where $\bar{p}(\boldsymbol{\Omega})$ and $\bar{p}(\mathbf{x})$ denote the corresponding thermal equilibrium probabilities. The noise $\xi(t)$ obeys the conditional average ${ }^{(32)}$ (fluctuationdissipation theorem of second kind)

$$
\left\langle\xi_{i}(t) \xi_{j}(0) \mid \mathbf{x}(t=0)=\mathbf{x}\right\rangle=k T \zeta_{i j}(\mathbf{x} ; t)
$$

This generalized Brownian motion dynamics, (2.5), forms the key input to a Kramers-like approach to the escape problem.

\subsection{Kramers Approach}

Kramers starts from the assumption that there is a clear-cut separation between the time scales of particle (mass $M$ ) and heat bath motions. Thus he applies a memoryless (Markovian) limit to the generalized Brownian motion dynamics in (2.5) with the noise being approximated by Gaussian white noise, i.e.,

$$
\begin{aligned}
\dot{x}=u, \quad \dot{u} & =-\frac{1}{M} \frac{\partial U}{\partial x}-\gamma u+\xi(t) \\
\langle\xi(t) \xi(s)\rangle & =(2 k T \gamma / M) \delta(t-s)
\end{aligned}
$$




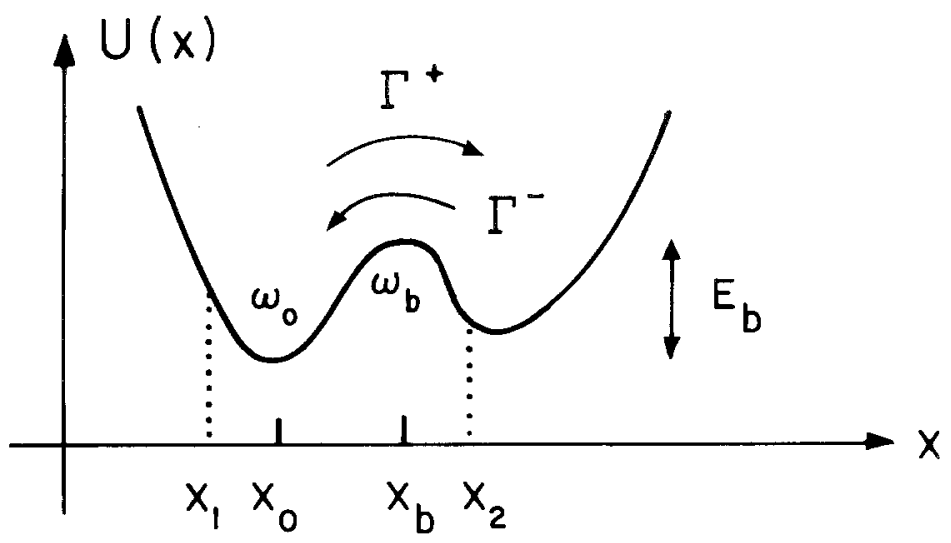

Fig. 1. Bistable potential field used in text. $E_{b}$ indicates the barrier factor for the thermally activated forward rate $\Gamma^{+}$.

Here, we have used a uniform momentum relaxation rate $(M \gamma)$. Moreover, we assume the motion to take place in a one-dimensional bistable potential field exhibiting two states of local stability (see Fig. 1). The Langevin dynamics in (2.6) is equivalent to an equation for the rate of change of probability $p_{t}(x, u)$ given by ${ }^{(1,34)}$ (Klein-Kramers equation)

$$
\begin{aligned}
\dot{p}_{t}(x, u)= & \left\{-u \frac{\partial}{\partial x}+\frac{\partial}{\partial u}\left[\gamma u+\frac{1}{M} \frac{\partial U(x)}{\partial x}\right]\right\} p_{t}(x, u) \\
& +\frac{\gamma k T}{M} \frac{\partial^{2}}{\partial u^{2}} p_{t}(x, u)
\end{aligned}
$$

This equation was derived first by Klein in 1922. If $U(x)$ is a nonlinear potential field, analytical results for (2.7) within the full damping regime are not known. For a cosine potential and a symmetric double well, numerical solutions for eigenfunctions and the generally complex-valued eigenvalues have been obtained only recently by Risken and coworkers. ${ }^{(35)}$ Analytical studies of the smallest relaxation eigenvalues are possible only in the limits of moderate-to-large friction ${ }^{(1-6,35)}$ and very small friction $\gamma^{(1,6 \mathrm{6}, 35 \mathrm{~b}, 36)}$ To evaluate the thermally activated escape rate, let us first assume that thermal equilibrium in the initial well is maintained at all times; i.e., the vertical thermalization (see Section 2.1) occurs on a sufficiently fast time scale such that deviations from the thermal Boltzmann probability $\vec{p}$ of (2.7) ( $Z$ : normalization),

$$
\bar{p}(x, u)=Z^{-1} \exp -\left[\frac{M}{2} u^{2}+U(x)\right] / k T
$$


inside the initial well can safely be neglected. Physically, this assumption holds whenever the damping strength is sufficiently large (moderate-tolarge friction regime; see Fig. 2); i.e.,

$$
\gamma \gtrsim \omega_{b} \equiv\left[\frac{1}{M}\left|U^{\prime \prime}\left(x_{b}\right)\right|\right]^{1 / 2}
$$

In this regime, the rate of escape is limited by collisions when the particle is near the top of the barrier. The frictional forces imply that a typical reaction path does not go directly from one side of the well to the neighboring well but rather may cross the barrier region many times, tottering back and forth before escaping.

In other words, within the reduced description there will now occur,--in contrast to the many-body-rate description in full phase space (Section 2.1)-many recrossings, which adequately can be described as a diffusive process over the col of the saddle point. If we were to apply the TST assumptions for this reduced, dissipative dynamics (2.6), each of the forward crossings on a single trajectory would be counted as contributing to the rate. Therefore, an equilibrium flux across the saddle point

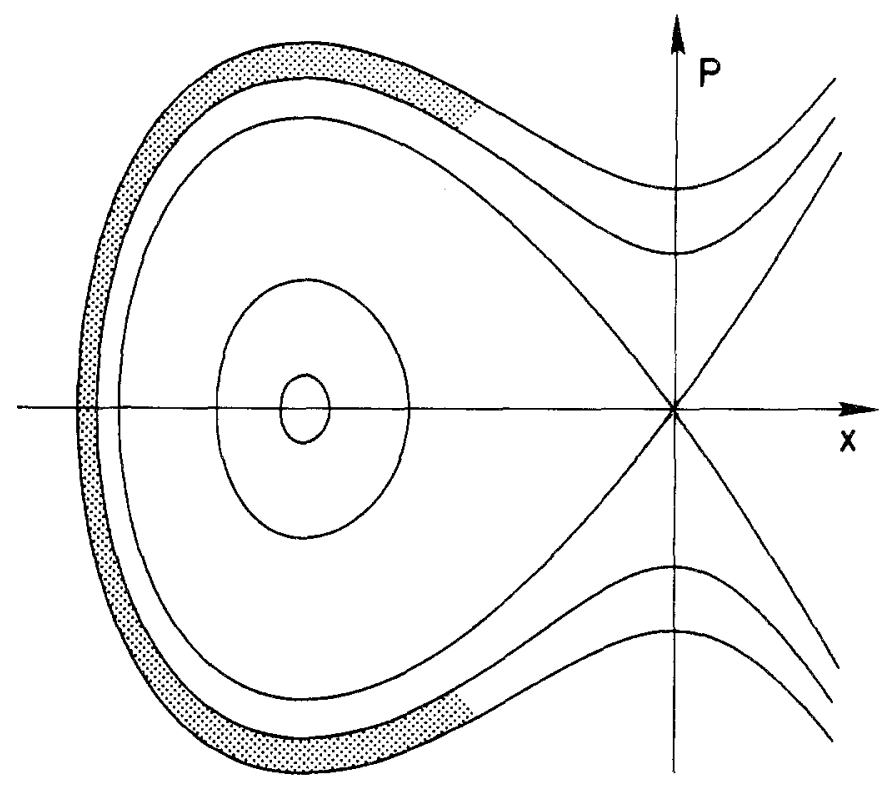

Fig. 2. Phase plane $(x, p=M u)$, with constant-energy contours. Dotted region shows the range over which derivation of (2.20) assumes thermal equilibrium. The nonequilibrium probability $p_{0}$ is falling off only as the barrier is approached (after Ref. $6 \mathrm{k}$ ). 
necessarily would overestimate the reaction rate $\Gamma$. In reality the diffusional crossings across the barrier will modify the Boltzmann distribution near the saddle point region, thereby yielding friction-induced deviations from thermal equilibrium near the barrier (see Fig. 2).

To evaluate this modified nonequilibrium probability $p_{0}$ we follow the original reasoning of Kramers. ${ }^{(1)}$ Considering the forward rate $\Gamma^{+} \equiv \Gamma$, let us inject particles near the well bottom around $x_{1}<x_{0}$ and assume that a particle sink is present to the right of $x_{b}$, around $x_{2}>x_{b}$. In the steady state, the so-induced stationary nonequilibrium probability, $p_{0}$, generates a flux $J_{0}$. If $n_{0}$ denotes the population of particles inside the initial well we obtain for the escape rate $\Gamma$ (flux-over-population approach)

$$
\Gamma=J_{0} / n_{0}
$$

If $\mathbf{x}$ denotes the set of reaction coordinates along the reaction path, (2.10) may be recast as ${ }^{(1-4)}$

$$
J_{0} \tau=\int_{\text {initial well }} p_{0}(\mathbf{x}) d x=n_{0}
$$

where $\tau \sim \Gamma^{-1}$ is the average time scale for escape. Thus our task consists in calculating this nonequilibrium probability $p_{0}$. Kramers uses in terms of the equilibrium probability $\bar{p}(x, u),(2.8)$, the ansatz

$$
p_{0}(x, u)=F(x, u) \bar{p}(x, u)
$$

in which $F(x, u)$ obeys (within our assumption of thermal equilibrium in the initial well) $F(x, u)=1$ around $x \sim x_{0}$ and $F(x, u)=0$ around $x \sim x_{2}$, near the sink. Clearly, all the action of the classical escape process takes place near the parabolic barrier. Hence, we can linearize the Klein-Kramers equation around $x=x_{b}$. Setting $y \equiv x-x_{b}$, we arrive at a relation for $F(y, u)$ :

$$
u \frac{\partial F}{\partial y}+\omega_{b}^{2} y \frac{\partial F}{\partial u}=\frac{k T \gamma}{M} \frac{\partial^{2} F}{\partial u^{2}}-\gamma u \frac{\partial F}{\partial u}
$$

Kramers solved (2.13) by use of the ansatz

$$
F(y, u) \equiv F(\eta)=F(u-c y)
$$

obeying the boundary condition (see above) $F(\eta) \rightarrow 1$ as $\eta \rightarrow+\infty$ and $F(\eta) \rightarrow 0$ as $\eta \rightarrow-\infty$. 
Upon substituting this ansatz into (2.13) one obtains

$$
-\left[(c-\gamma) u-\omega_{b}^{2} y\right] \frac{d F}{d \eta}=\frac{k T \gamma}{M} \frac{d^{2} F}{d \eta^{2}}
$$

In order for this to be consistent with (2.14), we must have

$$
\omega_{b}^{2}=(c-\gamma) c
$$

i.e.,

$$
-(c-\gamma) \eta \frac{d F}{d \eta}=\frac{k T \gamma}{M} \frac{d^{2} F}{d \eta^{2}}
$$

This relation is readily integrated to give

$$
F(\eta)=F_{0} \int^{\eta} \exp -\left[(c-\gamma) z^{2} /(2 k T \gamma / M)\right] d z
$$

To satisfy the boundary conditions we must use the positive root of (2.16); i.e.,

$$
c=\frac{\gamma}{2}+\left(\frac{\gamma^{2}}{4}+\omega_{b}^{2}\right)^{1 / 2}
$$

yielding

$F(\eta)=\left(\frac{c-\gamma}{2 \pi k T \gamma / M}\right)^{1 / 2} \int_{-\infty}^{\eta} \exp \left[-(c-\gamma) z^{2} /(2 k T \gamma / M)\right] d z$

The diffusive flux $J_{0}$ across the barrier is now readily determined

$$
J_{0}=\int_{-\infty}^{\infty} p_{0}\left(x=x_{b}, u\right) u d u
$$

which after an integration by parts yields

$$
=\left[\left(\frac{\gamma^{2}}{4}+\omega_{b}^{2}\right)^{1 / 2}-\frac{\gamma}{2}\right] \frac{k T}{Z \omega_{b} M} \exp \left[-U\left(x_{b}\right) / k T\right]
$$

For the population inside the initial well we obtain with $F(x, u) \simeq 1$

$$
n_{0}=\frac{2 \pi k T}{Z \omega_{0} M} \exp \left[-U\left(x_{0}\right) / k T\right], \quad \omega_{0}^{2}=\frac{1}{M} U^{\prime \prime}\left(x_{0}\right)
$$


Combining (2.18) and (2.19), we arrive at Kramers' expression for the thermally activated escape rate, valid in the moderate-to-large friction regime $^{(1), 6}$

$$
\Gamma=\left(\frac{\mu}{\omega_{b}}\right) \frac{\omega_{0}}{2 \pi} \exp \left(-E_{b} / k T\right)
$$

Therein, $E_{b}=U\left(x_{b}\right)-U\left(x_{0}\right)$, and

$$
\mu=\left[\left(\frac{\gamma^{2}}{4}+\omega_{b}^{2}\right)^{1 / 2}-\frac{\gamma}{2}\right]<\omega_{b}
$$

$\mu$ is the friction-induced (angular) transmission frequency. We may define a diffusive transmission factor $\kappa=\mu / \omega_{b}<1$ (effects of recrossings), and recast the prefactor $A$ in (2.20a) as

$$
A=\kappa \frac{\omega_{0}}{2 \pi} \equiv v^{*}
$$

Actually, this may be interpreted as the corresponding, effective many-body TST frequency $v^{*},(2.4)$, which would have resulted if we had not performed the (Markovian) reduction of the phase space dynamics of system plus environment. In the overdamped limit, the result in (2.20) simplifies further to give the well known result ${ }^{(1)}$

$$
\Gamma^{\text {overdamped }}=\frac{\omega_{0} \omega_{b}}{2 \pi \gamma} \exp \left(-E_{b} / k T\right), \quad \gamma \gg \omega_{b}
$$

which approaches zero as $\gamma \rightarrow \infty$.

Alternatively, we can obtain the result in (2.22) by contracting further the dynamics in (2.6) onto a single coordinate variable; i.e., $(x, u) \rightarrow x$, and then taking the strongly overdamped limit. An exact reduction of (2.6) onto a single variable flow, $\dot{x}$, results for the rate of change of propability $p_{t}(x)$ in a non-Markovian description of rather complex form, exhibiting, memory as well as an integral-operator structure. The study of approximate reductions of the Klein-Kramers equation has attracted a great deal of attention in recent years within the general theme of "adiabatic elimination procedures."(37) In the strongly overdamped limit this procedure again yields a Fokker-Planck description, widely known as the Smoluchowski equation. ${ }^{(38)}$ It is valid on time scales long compared to

\footnotetext{
${ }^{6}$ See Eq. (25) in Ref. 1. Note that Kramers uses the symbol $\omega$ to denote not the angular fre-
} quency, $\omega=2 \pi v$, but the frequency $v$ itself. 
$\gamma^{-1}$ and whenever neither the potential, $U(x)$, nor the force, $U^{\prime}(x)$, vary appreciable on a length scale $l=\left(k T / M \gamma^{2}\right)^{1 / 2}$; i.e.,

$$
l\left|\frac{\partial U}{\partial x}\right| \ll k T, \quad l\left|\frac{\partial^{2} U}{\partial x^{2}}\right| \ll\left|\frac{\partial U}{\partial x}\right|
$$

If we allow also for a coordinate-dependent position-diffusion coefficient $D(x)$; i.e., $D=k T /(M \gamma) \rightarrow k T /[M \gamma(x)]$, we have for the Smoluchowski equation the result

$$
\dot{p}_{t}(x)=\left[\frac{\partial}{\partial x} D(x)\left(\beta \frac{\partial U(x)}{\partial x}+\frac{\partial}{\partial x}\right)\right] p_{t}(x)
$$

where we have introduced the inverse temperature $\beta=(k T)^{-1}$. The rate of escape in a multistable potential field $U(x)$ can now be evaluated for this Smoluchowski dynamics just as before. We again inject particles near $x_{1}<x_{0}$ and remove them near $x_{2}>x_{b}$, thereby generating a stationary nonequilibrium current $J_{0} \neq 0$. The corresponding nonequilibrium probability $p_{0}(x)$ obeys

$$
J_{0}=-D(x)\left[\beta \frac{\partial U(x)}{\partial x}+\frac{\partial}{\partial x}\right] p_{0}(x)
$$

with the boundary conditions $p_{0}\left(x \sim x_{1}\right)=1$ and $p_{0}\left(x=x_{2}\right)=0$. Again setting

$$
p_{0}(x)=F(x) \bar{p}(x)
$$

one finds for the deviation from thermal equilibrium

$$
F(x)=-J_{0} \int_{x_{2}}^{x} \frac{d y}{\bar{p}(y) D(y)}
$$

Note that the nonequilibrium form factor $F(x)$ rapidly approaches a constant inside the well, $x<x_{b}$, and rapidly decreases for $x_{b}<x<x_{2}$. In terms of the population in the initial well

$$
n_{0}=\int_{x_{1}}^{x_{b}} F(x) \bar{p}(x) d x
$$

the escape rate emerges as

$$
\Gamma=\frac{J_{0}}{n_{0}}=\left[\int_{x_{1}}^{x_{b}} \vec{p}(x) d x \int_{x}^{x_{2}} \frac{d y}{\bar{p}(y) D(y)}\right]^{-1}
$$


By use of a steepest descent approximation this expression simplifies to give

$$
\Gamma=\frac{\omega_{0} \omega_{b}}{2 \pi \gamma\left(x_{b}\right)} \exp \left(\frac{-E_{b}}{k T}\right)
$$

which with $\gamma\left(x_{b}\right) \equiv \gamma$ precisely equals the previous result in (2.22).

\subsection{Alternative Methods}

The flux-over-population method of the previous section is a quite general approach to the evaluation of the rate of escape from a metastable state. However, there are other techniques which under certain circumstances might be used preferably. Most importantly, there is a connection between the escape rate and the smallest eigenvalue governing the time evolution of an initial nonequilibrium probability, or a stationary correlation function. Let $N_{A}(t)$ denote the fraction of particles with $x<x_{b}$ and $N_{B}(t)$, the fraction of particles with $x>x_{b}$, respectively, such that

$$
N_{A}(t)+N_{B}(t)=1
$$

In terms of the forward rate, $\Gamma^{+}$, and the backward rate, $\Gamma^{-}$(see Fig. 1), the kinetics can be described phenomelogically by a first-order rate law

$$
\begin{aligned}
& \dot{N}_{A}(t)=-\Gamma^{+} N_{A}(t)+\Gamma^{-} N_{B}(t) \\
& \dot{N}_{B}(t)=-\Gamma^{-} N_{B}(t)+\Gamma^{+} N_{A}(t)
\end{aligned}
$$

This, of course, leads to a relaxational dynamics for $N_{A}(t)$ [or $\left.N_{b}(t)\right]$

$$
N_{A}(t) \sim \exp \left[-\left(\Gamma^{+}+\Gamma^{-}\right) t\right]
$$

Owing to the clear-cut time separation between hopping dynamics and local relaxation to stable equilibrium, the relaxation rate, $\lambda$, of (2.29) can be identified with the smallest real part, $\operatorname{Re} \lambda_{1}$, of the generally complexvalued eigenvalues of dissipative operators ${ }^{(39)}$ of the type in $(2.7)$; i.e.,

$$
\lambda=\left(\Gamma^{+}+\Gamma^{-}\right) \simeq\left|\operatorname{Re} \lambda_{1}\right|
$$

In a symmetric double well one obtains

$$
\Gamma^{+}=\Gamma^{-}=\frac{1}{2}\left|\operatorname{Re} \lambda_{1}\right|
$$

More generally, for asymmetric wells

$$
\begin{aligned}
& \Gamma^{+} \simeq\left|\operatorname{Re} \lambda_{1}\right|\left(\frac{K}{K+1}\right) \\
& \Gamma^{-} \simeq\left|\operatorname{Re} \lambda_{1}\right|\left(\frac{1}{K+1}\right)
\end{aligned}
$$


where, $K=\Gamma^{+} / \Gamma^{-}$, denotes the equilibrium constant. In the presence of a finite asymmetry we have $U\left(x_{b}\right)-U\left(x_{0}\right) \neq U\left(x_{b}\right)-U\left(x_{0}^{\prime}\right)\left(x_{0}^{\prime}\right.$ : position of neighboring minimum), i.e., one of the escape rates is exponentially suppressed over the other.

There exist a variety of techniques for evaluating the smallest real part of the relaxation frequencies. In particular, we mention here the continued fraction method for correlation functions, ${ }^{(39,40)}$ the matrix-continued fraction method of Risken, ${ }^{(42)}$ variational methods, ${ }^{(6 \mathrm{~b}, 6 \mathrm{f})}$ the Laplace method of Skinner and Wolynes, ${ }^{(6 \mathrm{~g})}$ or the path-integral and instanton technique. ${ }^{(42)}$ An evaluation of $\lambda_{1}$ is particularly simple for dissipative operators which can be symmetrized. ${ }^{(39)}$ In this case, all eigenvalues are real and negative.

Another alternative to the flux-over-population method involves the concept of the mean first passage time. ${ }^{(43)}$ As is evident by the other articles in this proceedings, this concept has become very popular in recent years. We will not belabor here the recent progress in this field, ${ }^{(44-48)}$ but rather focus only on its connection with escape rates. The mean first passage time is a rather complex notion for a general stochastic process. ${ }^{(44-46,48)}$ For the problem of escape from a domain of attraction, asymptotic results have been evaluated in higher-dimensional Fokker-Planck systems. ${ }^{(44,45 d, 45 e, 46)}$

General exact results have only been obtained for one-dimensional Fokker-Planck processes ${ }^{(43-47)}$ and one-dimensional birth- and death-type processes. ${ }^{(6 e, 43,45 b, 45 c)}$ (The interested reader is directed to the very pedagogical discussion of one-dimensional, diffuse barrier crossing problems given by Schulten et al. ${ }^{(47)}$ )

Let us denote by $\tau(\bar{x})$ the mean of the first passage time variable of a random walker which starts at $\bar{x} \in \mathbf{I}$ at time $t_{0}=0$ and makes an exit from a specified interval I. With a one-dimensional Fokker-Planck dynamics in mind, let $x_{1}<x_{0}$ be a reflecting boundary, i.e., $\partial \tau(\bar{x}) /\left.\partial \bar{x}\right|_{\bar{x}=x_{1}}=0$, and $x_{2}>x_{b}$ be an absorbing boundary, or, $\tau\left(\bar{x}=x_{2}\right)=0^{(43)}$ (Fig. 1). Standard analysis then gives for $\tau(\bar{x})$, with $\bar{x}$ denoting a value less than the barrier position $x_{b}$, (escape time!)

$$
\frac{1}{\tau(\bar{x})}=\left[\int_{\bar{x}}^{x_{2}} \frac{d x}{D(x) \bar{p}(x)} \int_{x_{1}}^{x} \bar{p}(y) d y\right]^{-1}
$$

Note here the similarity between (2.33) and (2.26). This similarity is to be expected by alluding to $(2.11)$, where $\left(\Gamma^{+}\right)^{-1}$ is related to the "mean escape time," $\tau$, which in turn can be identified with $\tau(\bar{x})$ as given by $(2.33)$. Although different, both (2.33) and (2.26) yield upon a steepest descent approximation precisely the result in (2.27). For this identification to be valid, it is important to note that a correct choice of boundary conditions in (2.33) is essential in order for $\tau(\bar{x})$ to be of the order of the mean escape 
time $\tau$. For example, if we would have chosen instead that both $x_{1}<x_{0}$ and $x_{2}$ are absorbing, one obtains

$$
\begin{aligned}
\tau(\bar{x})= & \int_{\bar{x}}^{x_{2}} \frac{d x}{D(x) \bar{p}(x)} \int_{x_{1}}^{x} \bar{p}(y) d y \\
& -\int_{x_{1}}^{x_{2}} \frac{d x}{D(x) \bar{p}(x)} \int_{x_{1}}^{x} \bar{p}(y) d y\left[\int_{\bar{x}}^{x_{2}} \frac{d y}{D(y) \bar{p}(y)} / \int_{x_{1}}^{x_{2}} \frac{d y}{D(y) \bar{p}(y)}\right]
\end{aligned}
$$

In the steepest-descent approximation this gives

$$
\tau(\bar{x})=\frac{1}{\Gamma^{+}}\left[1-\int_{\bar{x}}^{x_{2}} \frac{d y}{D(y) \bar{p}(y)} / \int_{x_{1}}^{x_{2}} \frac{d y}{D(y) \bar{p}(y)}\right] \simeq O(1)
$$

which is obviously incorrect. This is readily understood if we note that (2.34) gives the average time for absorption (either at $x_{1}$ or $x_{2}$ ) in terms of the absorption time in (2.33), minus the probability that absorption occurs at boundary $x=x_{1}$, multiplied by the average absorption time in (2.33), when $x(t)$ starts out at (reflecting) boundary $x=x_{1}$. Thus, in situations where the construction of correct boundary conditions is difficult, as is the case for stochastic processes driven by shot noise (master equation dynamics $^{(48)}$ and non-Markovian processes ${ }^{(45 a, b, c)}$, one is probably better off with the flux-over-population method in Section 2.2.

The discussion of a third technique, the imaginary free energy method, will be deferred to Section 3, where we elaborate on the quantum treatment of the escape. Finally, for an illustrative discussion of the various interrelationships among Kramers' escape rate and the diffusion-controlled reaction scheme pioneered by Smoluchowski, ${ }^{(49)}$ Debye ${ }^{(50)}$ and Collins and Kimball, ${ }^{(51,52)}$ we refer the reader to an article by Shoup and Szabo. ${ }^{(53)}$

\subsection{Weakly Damped Systems}

In Section 2.2 we have addressed the Kramers approach for moderateto-large friction where the associated nonequilibrium effects can be modeled in terms of diffusional surface recrossings over the barrier. As emphasized earlier, there is generally a second type of nonequilibrium effect which is related to the deviation from thermal equilibrium inside the initial well. Such effects play an increasingly more important role for weak friction where the particle suffers infrequent collisions, i.e., the energy $E$ will be the only slowly relaxing variable. Two limiting cases have been discussed in the

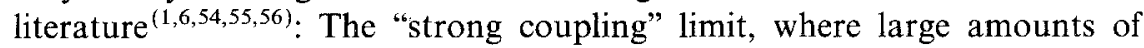
energy can be exchanged upon a collision, and the "weak coupling" limit, or low-friction Kramers (energy diffusion) limit. 
The strong coupling limit has attracted a good deal of attention among chemists seeking to model unimolecular gas phase reactions. ${ }^{(6 \mathrm{~g}, 27,54,65)}$ In this limit, internal equilibrium is maintained in the initial well below some threshold energy; above this threshold, however, there exist nonequilibrium effects due to perturbations by reactive losses. If we characterize the dynamics via a master equation in energy space with the transition kernel denoted by $\Gamma\left(E \rightarrow E^{\prime}\right)$, one obtains for the rate in the strong coupling limit ${ }^{(6 \mathrm{~g}, 54,65)}$

$$
\Gamma=\int_{0}^{E_{b}} \lambda(E) \bar{p}(E) / \int_{0}^{E_{b}} \bar{p}(E) d E
$$

where $\lambda(E)$ characterizes the collision frequency

$$
\lambda(E)=\int_{E_{h}}^{\infty} \Gamma\left(E \rightarrow E^{\prime}\right) d E^{\prime}
$$

Next let us focus on the weak coupling limit where only energy transitions small compared to $k T$ occur. ${ }^{(1)}$ This results in an average kinetic energy for the escaping particles at the barrier which approaches zero as the friction strength $\gamma$ goes to zero. ${ }^{(55)}$ Because the transition kernel $\Gamma\left(E \rightarrow E^{\prime}\right)$ is sharply peaked around $E \simeq E^{\prime}$, the dynamics now can be conveniently modeled by a Fokker-Planck equation in energy space [or alternatively in action space $J(E)$ with $d E / d J=v(E)],{ }^{(1)}$ i.e.,

$\dot{p}_{t}(E)=-\frac{\partial}{\partial E}\left[-D(E)\left(\frac{\partial}{\partial E}+\beta\right) \nu(E) p_{f}(E)\right], \quad \gamma \ll k T / J\left(E_{b}\right)$

with

$$
D(E)=v(E) D(J)=k T \gamma J(E)
$$

The evaluation of the escape rate proceeds along the lines outlined in (2.23)-(2.27) for the Smoluchowski case. Hence we assume a nonequilibrium probability $p_{0}(E)$

$$
p_{0}(E)=F(E) \bar{p}(E)
$$

where the equilibrium probability of $(2.38)$ reads

$$
\bar{p}(E)=\frac{Z^{-1}}{v(E)} \exp (-\beta E)
$$


With the boundary condition $p_{0}\left(E=E_{b}\right)=0$, we have

$$
F(E)=-J_{0} \int_{E_{b}}^{E} \frac{d E^{\prime}}{D\left(E^{\prime}\right) \exp \left(-\beta E^{\prime}\right)}
$$

Thus we arrive at an escape rate [see $(2.26)]$

$$
\Gamma=\left[\int_{0}^{E_{b}} \frac{\exp (-\beta E)}{v(E)} d E \int_{E}^{E_{b}} \frac{\exp (\beta y)}{D(y)} d y\right]^{-1}
$$

For deep wells, this result simplifies to

$$
\Gamma=\frac{\gamma J\left(E_{b}\right)}{k T} v_{0} \exp \left(-\beta E_{b}\right), \quad k T \gg \gamma J\left(E_{b}\right)
$$

which is precisely the result given in Kramers' 1940 paper. ${ }^{(1)}$

In conclusion, the escape rate for very weak damping, $\gamma \ll \omega_{b}$, is linearly proportional to the friction coefficient $\gamma$, and approaches zero as $\gamma \rightarrow 0$. Clearly, with $\gamma \ll \omega_{b}$, the motion inside the well is almost conservative and escape up the energy ladder becomes very difficult; i.e. there is no mechanism which could replenish the upper states $\left(E>E_{b}\right)$ once the first particles are gone. Note also that (2.43) can be expressed alternatively in terms of a mean-first passage time $\tau(\bar{x} \equiv E=0)$ :

$$
\Gamma=\frac{1}{\tau(E=0)}=\left[\int_{0}^{E_{b}} \frac{\exp (\beta E)}{D(E)} d E \int_{0}^{E} \frac{\exp (-\beta y)}{v(y)} d y\right]^{-1}
$$

In the approach outlined in (2.41)-(2.45), we have used for the deviation from thermal equilibrium below threshold $E<E_{b}$, a perfect sink at $E=E_{b}$; i.e., $p_{0}\left(E=E_{b}\right)=0$, which implies immediate horizontal depletion. More realistically, there are further deviations from thermal equilibrium also above threshold, induced by the horizontal outflow in the presence of weak but finite damping. The outflow above threshold (see Fig. 3) is being compensated by a divergence in the vertical flux up in energy. This important extension, originally put forward by Buttiker, Harris, and Landauer, ${ }^{(6 j)}$ implies now that $p_{0}\left(E=E_{b}\right)>0$. It turns out that with weak friction $\gamma$, this particular change of boundary condition for $p_{0}\left(E_{b}\right)$ depends sensitively on the form of the potential. ${ }^{(6 j, 56,57)}$ In particular, it differs depending on whether the particles above threshold $E>E_{b}$ are allowed to escape to infinity or if they are allowed to "bounce back" from the walls of the confining potential in the neighbouring well (see Fig. 3). For an escape into the continuum one obtains for the rate ${ }^{(56)}\left(\gamma<\omega_{b}\right)$

$$
\Gamma=\left[\frac{\chi\left(E_{b}\right)-1}{\chi\left(E_{b}\right)+1}\right]\left[\int_{0}^{E_{b}} \eta(E) \frac{\exp (-\beta E)}{v(E)} d E \int_{k T}^{E_{b}} \frac{d y}{D(y) \exp (-\beta y)}\right]^{-1}
$$




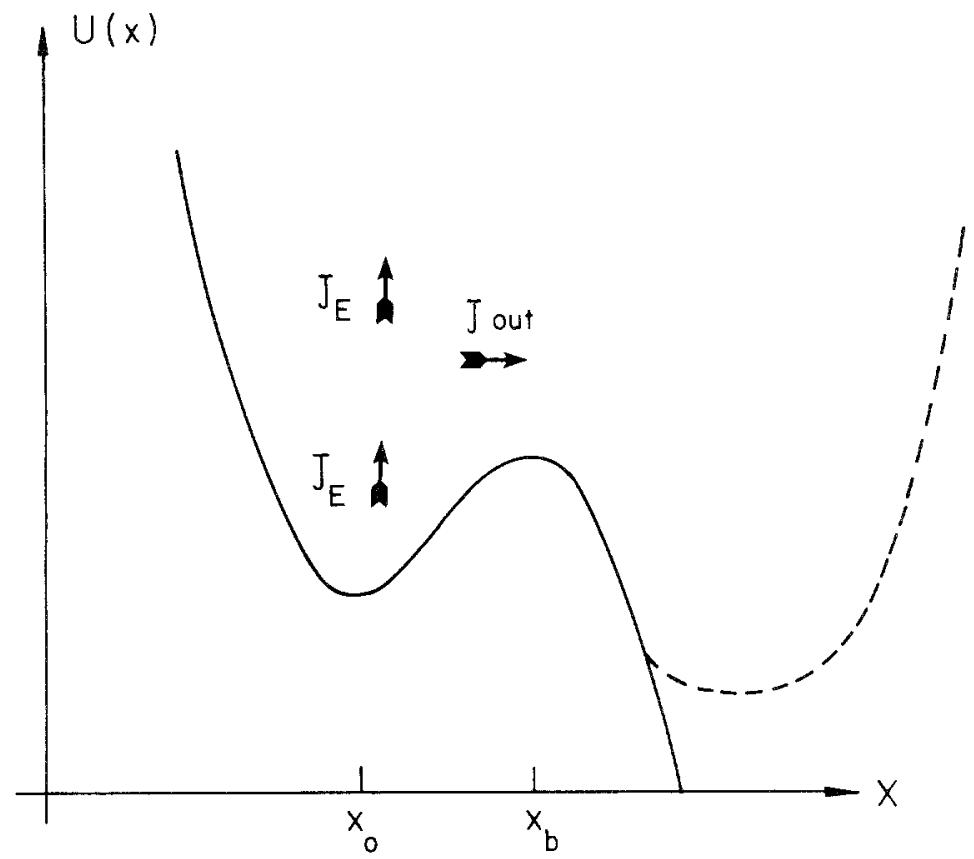

Fig. 3. Schematic sketch of the potential $U(x)$ assumed in text for the derivation of Eqs. (2.46)-(2.48). The diffusive vertical flux along the energy coordinate is $J_{E}$ and $J_{\text {out }}$ is a horizontal current giving the flux out of the well above the threshold energy. The dotted line indicates a potential form in which the particles can bounce back.

where at $E \simeq E_{b}$

$$
\eta(E) \simeq \frac{\exp \left(-\left\{\frac{1}{2}[\chi(E)-1] \beta\left(E-E_{b}\right)\right\}\right)}{\frac{1}{2}[\chi(E)+1]}
$$

and

$$
\chi(E)=\left[1+4(k T)^{2} / D(E)\right]^{1 / 2}
$$

For deep wells, (2.46) simplifies to ${ }^{(6 \mathrm{j}, 56) 7}$

$$
\begin{aligned}
\Gamma= & {\left[\frac{\chi\left(E_{b}\right)-1}{\chi\left(E_{b}\right)+1}\right] \frac{D\left(E_{b}\right)}{(k T)^{2}} v_{0} \exp \left(-\beta E_{b}\right) } \\
= & \left\{1-\gamma^{1 / 2}\left[J\left(E_{b}\right) / k T\right]^{1 / 2}+\frac{1}{2}\left[\gamma J\left(E_{b}\right) / k T\right]-\frac{1}{8}\left[\gamma J\left(E_{b}\right) / k T\right]^{3 / 2}+O\left(\gamma^{2}\right)\right\} \\
& \times \frac{\gamma J\left(E_{b}\right)}{k T} v_{0} \exp \left(-\beta E_{b}\right)
\end{aligned}
$$

${ }^{7}$ This result can be improved if we substitute in $\chi\left(E_{b}\right)$ the factor 4 by $4 \rightarrow 4 x$, where $x=1.474$ for a metastable potential (solid line in Fig. 3) and $\alpha=4.293$ for a symmetric double well ${ }^{(36)}$. In the latter case, the escape time $\tau$ also must be substituted by $\tau \rightarrow 2 \tau$, because the probability to bounce back equals $\sim \frac{1}{2}$. 
This weak friction rate exhibits a characteristic algebraic correction with a $\gamma^{3 / 2}$ dependence. Risken and Voigtlander ${ }^{(35 b, 36)}$ have found the same dependence on $\gamma$ in their study of the smallest eigenvalue, $\operatorname{Re} \lambda_{1}$, of the Klein-Kramers equation (2.7) at weak friction. The corresponding result, (2.47), for a symmetric double well can be found in Ref. 57. In contrast to the weak noise escape rates given in (2.20), (2.22), (2.27), valid for $\gamma \gtrsim \omega_{b}$, the rates at weak friction (2.44), (2.47) depend via the action $J\left(E_{b}\right)$ on the detailed shape of the potential field $U(x)$. Moreover, it should be noted that the differences between the deep-well approximations (2.44), (2.47) and the integral expressions in $(2.43,2.45,2.46)$ can be quite substantial for small barrier factors, $E_{b} / k T<6$.

\subsection{Multidimensional Metastability in Thermal Equilibrium}

So far we have addressed only the problem of escape in one-dimensional multistable potential fields (Sections 2.2-2.4). The results of Section 2.2 and 2.4 can readily be extended to escape in a multidimensional potential field $U\left(x_{1}, \ldots, x_{N}\right)=U(\mathbf{x}) .^{(3,4,5,44,45,46,58)}$ If transport of particles occurs over sequential saddle points our previous results will be modified by an entropy factor (see Section 2.1) which compares the number of states in the saddle point region with the number of states at initial metastable equilibrium. If the damping, $\gamma$, across the saddle point in the direction of steepest descent is in the moderate-to-large friction regime, we obtain ${ }^{(3-5)}$

$$
\Gamma=\frac{\mu}{\omega_{b}}\left(\frac{\prod_{i=1}^{N} v_{i}^{0}}{\prod_{i=1}^{N-1} \bar{v}_{i}}\right) \exp \left(\frac{-E_{b}}{k T}\right)
$$

or

$$
\Gamma=\frac{\mu}{\omega_{b}} \Gamma_{N}^{\mathrm{TST}}
$$

wherein $\mu$ is the friction-induced transmission frequency defined earlier in (2.20), and $\Gamma_{N}^{\mathrm{TST}}$ denotes the corresponding multidimensional generalization of simple TST. The set $\left\{v_{i}^{0}\right\}$ are the $N$ stable frequencies in the potential well and $\left\{\bar{v}_{i}\right\}$ are the $(N-1)$ stable frequencies at the saddle point.

Accordingly, in the weak friction regime the results in 2.4 can be generalized to Brownian motion in higher dimension if the corresponding $N$-dimensional generalization of the diffusion coefficient $D(E)$ is substituted into the formulas (2.38), (2.42), (2.43), (2.45), (2.46), (2.47). In terms of the hydrodynamic friction tensor $\zeta_{i j}(\mathbf{x})$, one obtains ${ }^{(59)}$

$$
D(E)=\sum_{i, j} \frac{k T}{m_{i} m_{j}} \frac{\left\langle\zeta_{i j}(\mathbf{x}) p_{i} p_{j}\right\rangle_{E}}{v(E)}
$$


with $\langle\cdots\rangle_{E}$ denoting the microcanonical average. ${ }^{(59)}$ For a configurationindependent friction tensor, this simplifies to

$$
D(E)=k T \gamma \frac{1}{\psi(E)} \int_{0}^{E} \psi\left(E^{\prime}\right) d E^{\prime} / v(E)
$$

where

$$
\gamma=\sum_{i=1}^{N} \frac{\zeta_{i i}}{m_{i}} \equiv \sum_{i=1}^{N} \gamma_{i i}
$$

is the mass-weighted friction tensor and $\psi(E)$ is the density of states

$$
\psi(E)=\int d \Omega \delta(E-\mathscr{H}(\mathbf{\Omega}))
$$

For example, let us consider an $N$-dimensional harmonic well with one oscillator mode truncated at $x_{b}$ at energy $E=E_{b}$. Then $\psi(E) \propto E^{N-1}$, and the rate becomes ${ }^{(59,60)}$

$$
\Gamma=\gamma \frac{\left(\beta E_{b}\right)^{N}}{N !} \exp \left(-\beta E_{b}\right)
$$

This result also provides a valuable working approximation for more general potential forms, provided that the barrier is sufficiently high and the motion near barrier threshold is completely irregular. ${ }^{(59)}$

\subsection{Kramers Theory with Memory Friction}

Although Kramers' landmark paper ${ }^{(1)}$ on the escape of a Brownian particle from a metastable state has found numerous applications, ${ }^{(2-21)}$ it lacks general applicability owing to the assumption of a clear-cut separation between the time scales of particle motion and heat bath motion; i.e., the particle must move slowly compared to rapid fluctuations exerted on the particle by the heat bath. However, in certain systems ${ }^{(7,14-19)}$ the relevant motion of the escape dynamics may take place on the same time scale or be even more rapidly than those used in measuring the static damping coefficients. Therefore memory effects of the type exhibited in the generalized Langevin equation (1.5) must be accounted for. Several recent experiments $^{(14-21)}$ involving classical thermal activation have shown a failure of the standard Kramers approach based on frequency-independent friction. This is due to the fact that in many situations the typical barrier frequency, $\omega_{b}$, is of the order $10^{11}-10^{14} \mathrm{sec}^{-1}$ and the environmental forces 
are likely to be correlated on this same time scale, thus giving rise to memory effects [i.e., $\left.\hat{\gamma}\left(\omega=10^{13} \mathrm{sec}^{-1}\right) \neq \hat{\gamma}(\omega=0)\right]$. We now extend Kramers' approach to multistable Brownian motion with arbitrary longtime memory. Here, we follow closely the reasoning of Mojtabai and Hanggi. ${ }^{(61)}$ First we will assume that thermal equilibrium inside the initial well is maintained at all times; i.e., the memory-renormalized friction is sufficiently large such that the position diffusion across the barrier presents the rate-dominating step. Linearizing the barrier dynamics in (2.5) in the variable $y=x-x_{b}$, we arrive at a diffusive dynamics given by

$$
\ddot{y}=\omega_{b}^{2} y-\int_{0}^{t} \gamma(t-\tau) \dot{y}(\tau) d \tau+\xi(t)
$$

Next, the thermal noise $\xi(t)$ is assumed to be stationary Gaussian noise (central limit theorem) obeying ${ }^{(32)}$

$$
\langle\xi(t) \xi(0)\rangle=k T \gamma(t) / M
$$

Because of the linear structure of $(2.55)$, the process $(y, \dot{y})$ is governed by a Gaussian non-Markovian process ${ }^{(39,62,63)}$ in an unstable $^{(61)}$ parabolic potential field. In terms of the time-convolutionless master equation ${ }^{(64)}$ we obtain for the rate of change of probability $p_{t}(y, u=\dot{y})$

$$
\begin{aligned}
\dot{p}_{t}= & {\left[-u \frac{\partial}{\partial y}-\bar{\omega}^{2}(t) y \frac{\partial}{\partial u}\right] p_{t}+\bar{\gamma}(t) \frac{\partial}{\partial u}\left(u p_{t}\right) } \\
& +\frac{k T}{M} \bar{\gamma}(t) \frac{\partial^{2}}{\partial u^{2}} p_{t}+\frac{k T}{M \omega_{b}^{2}}\left[\bar{\omega}^{2}(t)-\omega_{b}^{2}\right] \frac{\partial^{2}}{\partial u \partial y} p_{t}
\end{aligned}
$$

where

$$
\begin{aligned}
& \bar{\gamma}(t)=-\dot{a}(t) / a(t), \quad \bar{\omega}^{2}(t)=-b(t) / a(t) \\
& a(t)=\rho_{y}(t) \dot{\rho}_{u}(t)-\dot{\rho}_{y}(t) \rho_{u}(t), \quad b(t)=\dot{\rho}_{y}(t) \ddot{\rho}_{u}(t)-\ddot{\rho}_{y}(t) \dot{\rho}_{u}(t) \\
& \rho_{y}(t)=1+\omega_{b}^{2} \int_{0}^{t} \rho_{u}(s) d s, \quad \rho_{u}(t)=\mathscr{L}^{-1}\left[1 /\left(z^{2}-\omega_{b}^{2}+z \hat{\gamma}(z)\right],\right. \\
& \rho_{u}(0)=0
\end{aligned}
$$

Herein, $\hat{\gamma}$ denotes the Laplace transform of the memory friction $\gamma(t)$ and $\mathscr{L}^{-1}$ is the inverse Laplace transform. Evaluating the flux across the barrier, ${ }^{(61)}$ the final result for the rate emerges as ${ }^{(61,65)}$

$$
\Gamma=\left(\frac{\tilde{\mu}}{\omega_{b}}\right) \frac{\omega_{0}}{2 \pi} \exp \left(\frac{-E_{b}}{k T}\right)
$$


where $^{(61)}$

$$
\tilde{\mu}=\lim _{t \rightarrow \infty}\left\{\left[\frac{\bar{\gamma}^{2}(t)}{4}+\bar{\omega}^{2}(t)\right]^{1 / 2}-\frac{\bar{\gamma}(t)}{2}\right\}
$$

$\tilde{\mu}$ plays the role of a memory-renormalized diffusive transmission frequency (2.20b). Under fairly general conditions ${ }^{(66,67)}$ this memory-renormalized frequency equals the largest positive root of the relation ${ }^{(61,65-68)}$

$$
\tilde{\mu}=\frac{\omega_{b}^{2}}{\tilde{\mu}+\hat{\gamma}(\tilde{\mu})}
$$

appearing first in the work by Grote and Hynes (Ref. 65). It is rather amazing that this same expression, (2.59), reemerges in quite a different context $^{(69)}$ : It will be shown in Section 3 that $T_{0}=\hbar \tilde{\mu} /(2 \pi k)$ denotes the highest temperature below which the exponential Arrhenius factor in (2.58) ceases to describe the exponential leading part of the rate.

In applying the result in (2.58), some care should be taken. Just as in the memory-free situation, (2.20), the dynamic friction $\bar{\gamma}$ should be of sufficient strength such that the vertical thermalization inside the well occurs sufficiently rapidly. In this case, the effects due to nonequilibrium inside the metastable well and the effects due to the nonlinearities of the potential $U(x)$, important for moderate effective damping $\bar{\gamma}$, play a minor role. That is, in order to apply safely the relation in (2.58), we should have (in the limit $t \rightarrow \infty)^{8}$

$$
\begin{array}{ll}
\text { (i) } \bar{\gamma}>0, & \bar{\omega}>0 \\
\text { (ii) } \bar{\gamma} \gtrsim \bar{\omega} &
\end{array}
$$

Keeping the noise strength $\gamma_{0}$

$$
\gamma_{0} \equiv \lim _{\tau_{c} \rightarrow 0} \hat{\gamma}(z=0)
$$

a constant, successive increases of the memory correlation time $\tau_{c}$ tend to decrease the effective friction ${ }^{(69)}$ such that $\tilde{\mu}>\mu$ with $\mu,(2.20)$, evaluated in terms of $\gamma_{0}$. In other words, very strong memory correlation lowers the friction $\bar{\gamma}$ toward the smaller values of the energy-diffusion controlled regime. Actually, $\bar{\gamma}$ even takes on negative values for very large $\tau_{c}$. For moderate friction values, $\bar{\gamma} \approx \bar{\omega}>0$, the rate formula in (2.58) will be influenced by additional effects such as deviations from thermal equilibrium inside the

${ }^{8}$ In terms of the first two largest roots of (2.59), i.e., $\tilde{\mu}>\lambda_{1}>\lambda_{2} \ldots$, one obtains $\bar{\gamma}(\infty)=-\left(\tilde{\mu}+\lambda_{1}\right)$ and $\bar{\omega}^{2}(\infty)=-\tilde{\mu} \lambda_{1}$ (see also Appendix A in Ref. 67). 
initial well and horizontal, energy-diffusion controlled depletion effects (Section 2.4) which, in addition, depend sensitively also on the potential form (e.g., confining versus nonconfining potential fields; see Fig. 3). Moreover, (2.58) inherently reflects the result of a multidimensional steepest-descent approximation. That is, the positive-valued normal mode frequencies occurring in the minimum region and the saddle point region of an enlarged, Markovian stochastic description which models the memory friction ${ }^{(61 \mathrm{~b}, 68)},(2.55)$, should not contain pathological small relaxation frequencies which would invalidate the Gaussian approximations. If effects of this sort are present, the bona fide use of the rate formula (2.58) would clearly become questionable. Actually, some recent computer simulations ${ }^{(70)}$ in a symmetric double well and exponentially correlated noise exhibit trouble of precisely this sort for certain parameter regimes, with the criteria in $(2.60)$ being strongly violated.

In the regime of strong overdamping, $\bar{\gamma} \gg \bar{\omega}$, one obtains from (2.59)

$$
\tilde{\mu} \simeq \frac{\omega_{b}^{2}}{\hat{\gamma}(\tilde{\mu})} \approx \frac{\omega_{b}^{2}}{\hat{\gamma}(z=0)}
$$

Hence, the rate in (2.58) takes on the form of an overdamped Smoluchowski rate $(2.22)$ with $\gamma$ substituted by $\hat{\gamma}(z \approx 0)$. In certain situations, $\hat{\gamma}(z=0)$ exhibits a fractional power law dependence on transports coefficients, e.g., a viscosity, thereby giving rise to novel rate laws. ${ }^{(14,15,66)}$

For very weak damping, the influence of memory can be incorporated rather conveniently by evaluating the memory renormalized energy-diffusion coefficient which enters the effective energy-diffusion equation ${ }^{(71,72)}$ (2.38), with $D(E)$ replaced by the non-Markovian result ${ }^{(72 \mathrm{~d})}$

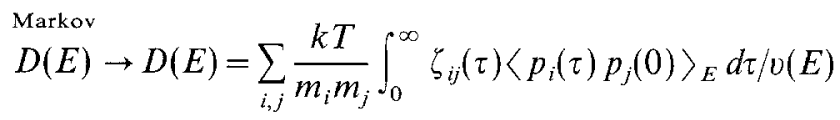

In one dimension this reduces to ${ }^{(72)}$

$$
\begin{aligned}
D(E) & =\frac{k T}{M} \int_{0}^{\infty} \gamma(\tau)\langle p(\tau) p(0)\rangle_{E} d \tau / v(E) \\
& =k T J(E) \int_{0}^{\infty} \gamma(\tau) \frac{\langle p(\tau) p(0)\rangle_{E}}{\left\langle p^{2}\right\rangle_{E}} d \tau
\end{aligned}
$$

where $\left\{p_{i}(\tau)\right\}$ are the deterministic, unperturbed momenta at energy $E$. The energy-diffusion controlled rate is then given by our previous results (2.43), (2.45), (2.46), (2.47). ${ }^{(56,72)}$ The diffusion coefficients in (2.62a), 
(2.62b) are dependent on potential form and also exhibit a notable dependence on the memory correlation time $\tau_{c{ }^{*}}{ }^{(72)}$ Because the non-Markovian diffusion coefficient is smaller than its Markovian counterpart, i.e.,

$$
D(E)<k T J(E) \int_{0}^{\infty} \gamma(\tau) d \tau
$$

there occurs a memory-induced decrease of the prefactor with increasing memory correlation $\tau_{c \cdot}{ }^{(56)}$

While the calculation of the escape rate $\Gamma$ covering the whole damping regime is very difficult, ${ }^{(56,6 \mathrm{k}, 67,73)}$ a rough uniform working approximation to the rate, $\Gamma^{\mathrm{UNIF}}$, in presence of general memory damping $\gamma(\tau)$ is obtained by writing ${ }^{(56)}$

$$
\Gamma^{\mathrm{UNIF}} \simeq\left[\tau\left(E_{b}\right)+\left(\frac{\omega_{b}}{\tilde{\mu}} / \Gamma_{N}^{\mathrm{TST}}\right)\right]^{-1}
$$

with $\tilde{\mu}$ given by $(2.58),(2.59)$, and $\tau\left(E_{b}\right)$ denotes the average energy-diffusion controlled escape time determined by (2.62), (2.43), (2.45), or (2.46). Deviations from this simple estimate most likely occur for strong memory correlation (weak-to-moderate effective friction regime). In potential fields which allow for substantial backflow, we must substitute $\tau\left(E_{b}\right) \rightarrow 2 \tau\left(E_{b}\right)$.

\subsection{Bistable Flows in Driven Systems}

The problem of noise-induced escape can also be broadened to treat the dynamics in driven systems within which multiple stable states can coexist, and transitions between these states being triggered by nonequilibrium noise sources. In the previous sections we focused on the escape from an equilibrium state driven by thermal noise. Of equal interest, however, are questions about the fluctuations and instabilities in nonequilibrium states. Indeed, a considerable effort has gone into the study of the fluctuations about such states. ${ }^{(39,41,74-76)}$ The investigation of such systems has been pioneered by Stratonovich ${ }^{(77)}$ and Landauer, ${ }^{(78)}$ who were also among the first to point out the analogies between equilibrium transitions of first-or second-order type and instabilities in driven systems. This notion has resurfaced more recently in the study of nonequilibrium transitions in nonlinear optical systems ${ }^{(79,80)}$ (laser and optical bistability). The ability to evaluate escape rates is important not only in itself but is also crucial for the determination of the relative stability of different stable states, including nonequilibrium states such as limit cycles and strange attractors. Examples of physical interest are the rate enhancement induced 
by parametric fluctuations in nonlinear oscillators, ${ }^{(81)}$ resonantly activated rate processes, ${ }^{(82)}$ transitions in externally synchronized oscillators, ${ }^{(77,83,84)}$ transport in sine-Gordon chains, ${ }^{(85)}$ the transitions between several configurations in a driven Josephson junction (or driven pendulum) such as the switching among locked-locked states at high damping ${ }^{(77,83,86)}$ or between locked-running (or vice versa) states at low damping, ${ }^{(6 j, 87)}$ transitions in optical bistability ${ }^{(45,46,80)}$ and transport in superionic conductors and charge density wave systems ${ }^{(88)}$ to name but a few.

The investigation of stationary nonequilibrium states is generically beset with difficulties which are absent in thermal equilibrium. In particular, there is the lack of detailed balance ${ }^{(89)}$ and the presence of drift fields which are not derivable from a potential field. Actually most of the multidimensional nonequilibrium systems do not possess a limiting weak noise, continuous differentiable thermodynamic potential $U(x)$, unless there exists a Hamiltonian system $H$, associated with the weak noise dynamics of the driven system, which is integrable on an $n$-dimensional separatrix of the $(2 n-1)$-dimensional energy hypersurface $H=0 .{ }^{(90)}$

The archetype of a bistable system beset with such trouble is the flow in a symmetric double well driven by colored, Gaussian noise of finite correlation $^{(91)}$

$$
\begin{aligned}
\dot{x} & =a x-b x^{3}+\xi(t), \quad a>0, b>0 \\
\langle\xi(t) \xi(s)\rangle & =\frac{D}{\tau_{c}} \exp \left(\frac{-|t-s|}{\tau_{c}}\right)
\end{aligned}
$$

This flow is equivalent to a two-dimensional Fokker-Planck dynamics which does not obey detailed balance. ${ }^{(91)}$ Keeping the noise strength $D$ fixed, one finds that the rate for (2.65) undergoes a characteristic exponential decrease upon increasing the memory correlation time $\tau_{c}{ }^{(91)}$ from its maximal value in the Smoluchowski limit (limit $\tau_{c} \rightarrow 0$ ) (Fig. 4). A similar exponential decrease for the rate upon increasing the memory correlation time $\tau_{c}$ has been found ${ }^{(92)}$ in systems driven by telegraphic noise (a twostate Markov process). In those systems one can study both the influence of finite memory and a non-Gaussian noise source (shot noise). ${ }^{(92)}$ In many cases, it is possible to reduce the nonequilibrium dynamics to a reduced, approximate single-variable Fokker-Planck dynamics, which intrinsically satisfies detailed balance. If justified, our task is simplified considerably in that the stationary probability $\tilde{p}$ is readily obtained by quadratures. Likewise, the current-carrying nonequilibrium probability $p_{0}(x)$

$$
p_{0}(x)=F(x) \bar{p}(x)
$$




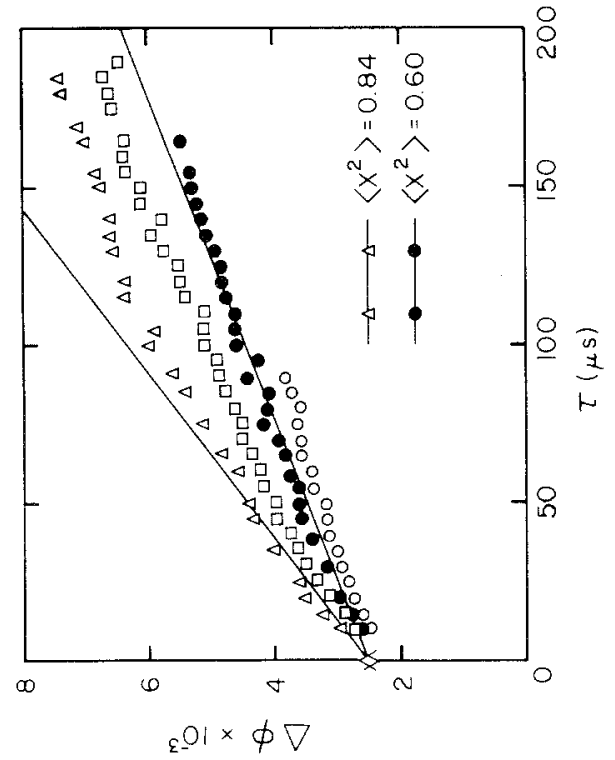

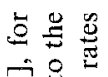

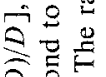

जी क्षि

青它

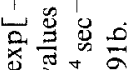

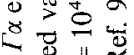

$-\bar{E} \|$

舟国

웜

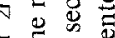

引吉苍

से

急 $\Xi$

힝

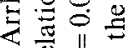

용용

$\sigma=0$

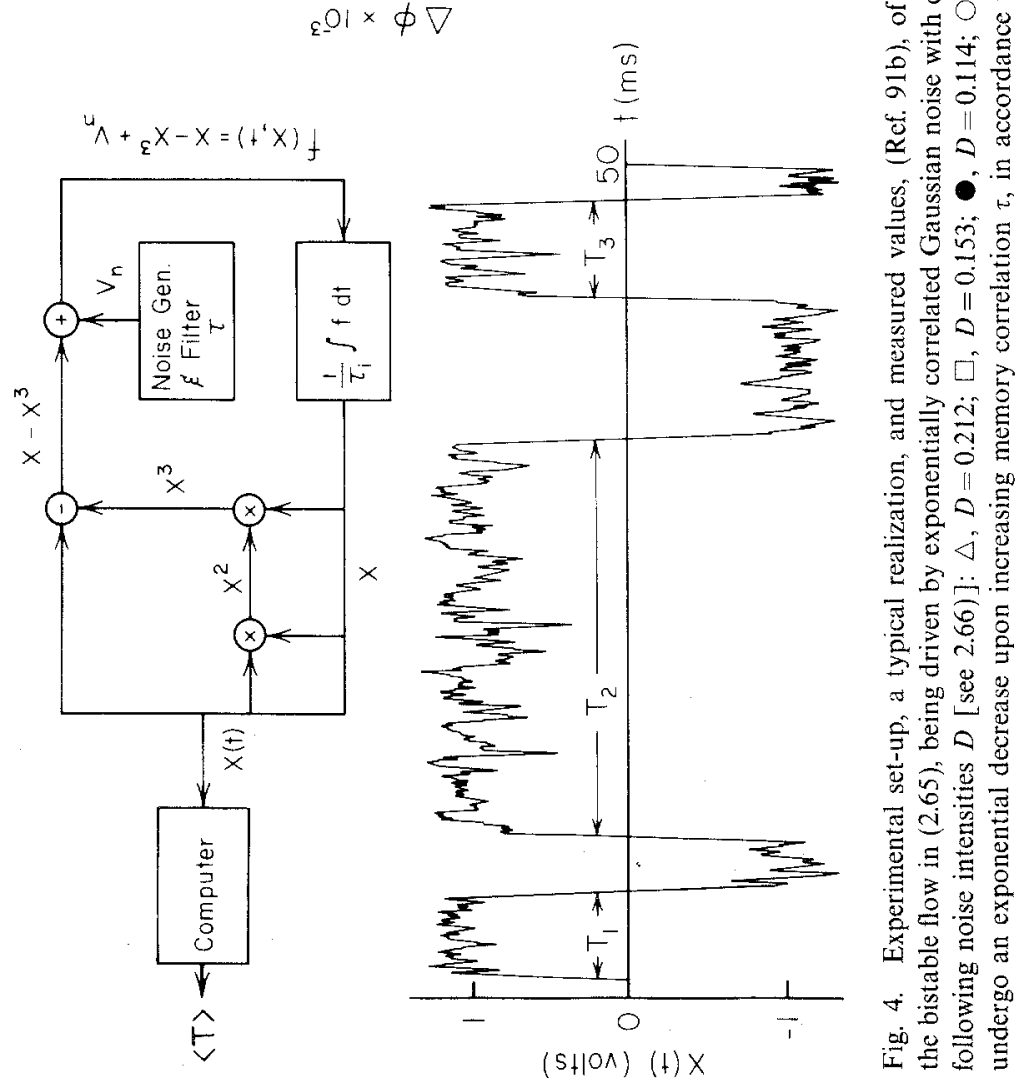


is obtained by use of the methods outlined in Section 2.2 for the overdamped situation (Smoluchowski equation). This is of particular importance in the context of recent progress, ${ }^{(93)}$ which shows that the long-time dynamics of a master equation dynamics can be modeled by a novel Fokker-Planck approximation yielding identical stationary probabilities, identical limiting weak noise escape rates, and identical decay times for relaxation from initial unstable states. ${ }^{(93)}$ Moreover, it has been demonstrated ${ }^{(93,94)}$ that a truncated Kramers-Moyal approximation (at second order) to a given shot-noise dynamics exponentially overestimates the escape rates.

\section{QUANTUM THEORETICAL TREATMENT}

\subsection{Historic Background and Perspectives}

In the previous sections, the general theme of thermal activated escape focused on a temperature regime in which quantum mechanical corrections could be neglected. At lower temperatures, however, the initial metastable state is rendered unstable progressively by quantum tunneling processes, as thermally activated processes become increasingly rare. Wigner ${ }^{(95)}$ and Bell $^{(96)}$ were the first to be concerned with an introduction of quantum corrections into the classical picture. Their work dealt mainly with the effects of thermally averaged transmission coefficients for inverted, untruncated parabolas at temperatures $T \gg \hbar \omega_{b} / 2 \pi k$. This early work, as well as later work, ${ }^{(97)}$ is based on a classical description of the jumping process in the presence of quantum statistical mechanics. The most advanced treatment along those lines is the work by Affleck, ${ }^{(98)}$ in which quantum statistical metastability of a single particle is treated at zero damping over the whole temperature regime $T \geqslant 0$ (simple quantum transition state theory). If the escaping particle is allowed to couple to environmental degrees of freedom such as phonons, magnons, and the like, thereby giving rise to quantum damping, the quantum theoretical treatment becomes a rather delicate challenge. The phenomenon of quantum tunneling in the presence of phonon modes is clearly ubiquitous in solid state physics. Early studies of phonon effects on tunnelling include those by Pirc and Gosar, ${ }^{(99)}$ Sander and Shore, ${ }^{(110)}$ and Sussmann. ${ }^{(101)}$ Actually, some of those authors seem to have been unaware of the relevance of Holstein's ${ }^{(102)}$ early multiphonon treatment (polaron problem) of this problem. Further developments of the polaronlike approach to quantum tunneling problems in the presence of phonon couplings include those by Flynn and Stoneham, ${ }^{(103)}$ Hopfield, ${ }^{(104)}$ Phillips, ${ }^{(105)}$ and Riseborough. ${ }^{(106)}$ Unfortunately, all of those elaborate theories relied upon the Condon approximation, i.e., that the tunneling matrix element $\Delta$ is independent of the phonon coordinates. The 
merits and demerits of those previous works are discussed in Sethna's beautiful articles. ${ }^{(107)}$ on zero temperature decay rates of tunneling centers, wherein he has adopted the imaginary time path-integral formalism (see below).

Recently, there has been a resurgence of interest in the effects that a thermodynamic bath has on the motion of a quantum mechanical particle, triggered by the possibility of observing macroscopic quantum tunneling phenomena in a medium with ohmiclike dissipation. ${ }^{(108,109)}$ The interest in this subject matter is due to the profound effect that this coupling produces, as well as the enormous variety of physical phenomena in which these processes occur. Caldeira and Leggett ${ }^{(109)}$ have investigated the influence of the thermal reservoir on the zero temperature quantum tunneling rate, using Feynman's functional integral formulation. ${ }^{(110)}$ In particular, they found that for ohmiclike dissipation the decay rate is strongly suppressed as compared to undamped systems. ${ }^{(109)}$ Likewise, Grabert, Weiss, and Hanggi ${ }^{(111)}$ have shaped this approach into an effective method which enables the study of various profound effects induced by finite temperature fluctuations in the range from $T \simeq 0$ up to the classical regime.

The path-integral approach is quite convenient because it allows the inclusion of the effect of the environment (heat bath) as an influence functional in much the same way as in Feynman's theories of the polaron and quantum noise. ${ }^{(112)}$ After integrating out the normal modes of the heat bath, the motion of the quantum particle is governed by an effective Lagrangian with a nonlocal term. One then proceeds by applying a field theoretic method which originally was invented by Langer ${ }^{(113)}$ in a study of classical nucleation theory. Coleman and Callan ${ }^{(114)}$ draw heavily on Langer's picture of classical nucleation theory, and beautifully popularized this technique for which they have coined the name "bounce method." The bounce trajectories studied in Refs. 109, 111, 115-117, are saddle points of a nonlocal action which inherently incorporates both the mass renormalization and the potential renormalization discussed in Section 2.1. These bounces bear a close analogy to the critical nucleus in the classical problem $^{(85,113)}$ in the sense that the bounce action plays the role of the energy of the critical droplet. If we were to proceed from the exact, nonlocal action without further approximation, all of the difficulties associated with such questions as adiabatic versus nonadiabatic transitions, ${ }^{(7)}$ the Born-Oppenheimer approximation, or the Condon approximation, would simply vanish. The traditional bounce technique, however, treats these problems with quasiclassical accuracy only, i.e., to lowest order in Planck's constant $\hbar$. This efficient bounce method has sometimes been met with skepticism, mainly due its arcane treatment of zero or even negative quantum fluctuation eigenvalue modes. Notably, this bounce method can be 
shown to be equivalent to a multidimensional WKB approximation in full phase space of system plus environment. ${ }^{(118,119)}$ In principle, the usual approximation inherent in the bounce technique, which amounts to the treatment of bounces via a dilute gas, can be improved further by considering also the fluctuation modes around multiple-bounce solutions (higher-order WKB approximation).

In the following I restrict the discussion to a quantum particle which is coupled to boson modes only. Moreover, I confine myself to the discussion of the quantum treatment of Kramers' escape problem into a continuum (see Fig. 5), and in addition assume that thermal equilibrium persists in the initial well at all times. Thus this amounts to a quantum treatment of the Kramers problem discussed in Sections 2.2, 2.5, and 2.6 over the whole temperature regime including $T=0$. From the physics point of view, this quantum version would then be equivalent with the quantum many-body transition state theory for a system coupled to $\sim 10^{23}$ environmental degrees of freedom.

For a discussion of some related, interesting problems such as the quantum decay into a continuum at weak bias ${ }^{(120)}$ and the energy loss dur-
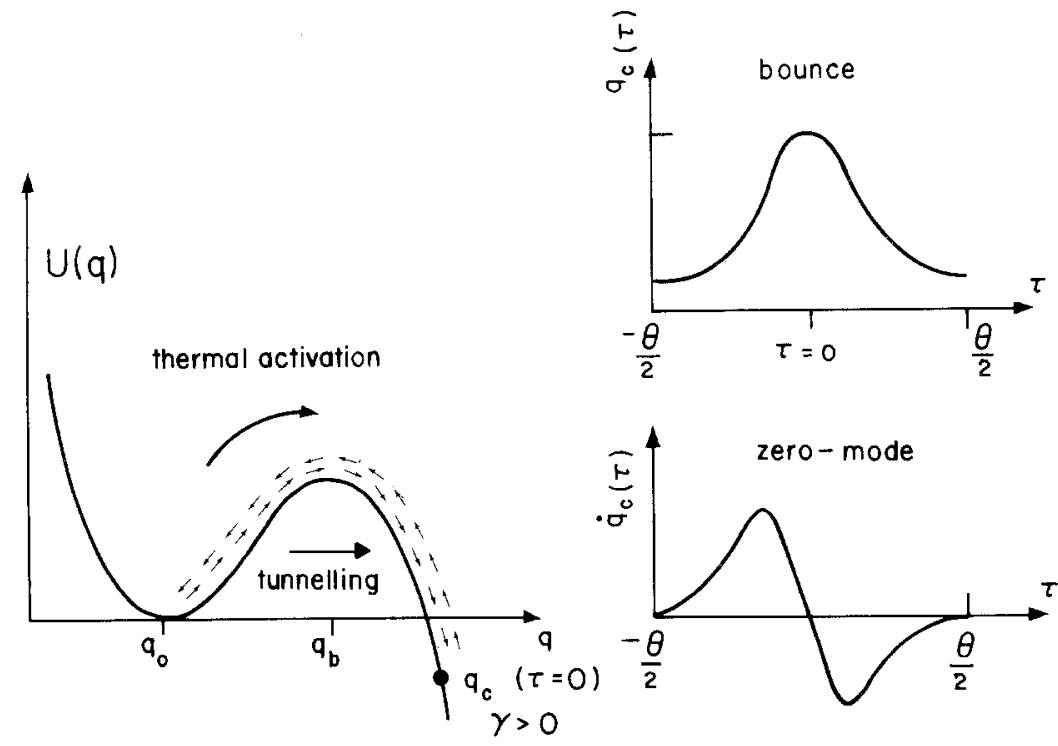

Fig. 5. Potential field used for evaluation of the quantum version of the Kramers rate. The periodic bounce trajectory, $q_{c}(\tau)$, which describes finite temperature quantum tunneling under the barrier, is a stationary point of the imaginary-time action; i.e., it obeys a classical equation of motion in the inverted potential $-U(q)$. The Goldstone mode, $\sim \dot{q}_{c}(\tau)$, possesses one node; thus there exists a fluctuation mode with negative eigenvalue. The point marked $q_{c}(\tau=0)$ indicates the zero-temperature bounce point in presence of finite dissipation $\gamma$. This then gives rise to a finite energy loss during tunneling (Ref. 120). 
ing tunneling, ${ }^{(120)}$ the quantum relaxation at finite temperatures in a symmetric double well ${ }^{(121)}$ or in a weakly biased double well ${ }^{(122)}$ we refer the reader to the original literature. Moreover, a discussion of our preliminary results $^{(123)}$ of a quantum version of Kramers' theory for weakly damped systems (see Section 2.4) is beyond the scope of this report.

\subsection{Simple Quantum Transition State Theory}

First, let us consider a particle of mass $M$ moving in a one-dimensional potential $U(q)$ (Fig. 5). We also assume that the particle is in contact with a heat bath at temperature $T$. However, the coupling is presumed to be infinitesimal so that the particle motion proceeds undamped $(\gamma=0)$. In other words, we consider the quantum transition state theory for a single particle moving in a one-dimensional potential $U(q)$. Therefore, for the results to be physically valid, one must continuously prepare an equilibrium distribution of particles in the initial well, e.g., with a Maxwell demon. Following the reasoning in Ref. 115, the decay rate $\Gamma$ is given by the imaginary part $(\mathrm{Im})$ of the space-diagonal Green's function $G$ over periodic paths with period $\theta$,

$$
\begin{aligned}
G(q, q ; \theta) & =\left\langle q\left|\exp \left[-i \theta\left(\mathscr{H}-\frac{i \hbar \Gamma}{2}\right) / \hbar\right]\right| q\right\rangle \\
& =\int_{q(0)=q(\theta)=q} \mathscr{D} q(t) \exp (i S[q(t)] / h)
\end{aligned}
$$

where $\theta=h / k T$ is the fundamental Matsubara period and $S[q(t)]$

$$
S[q(t)]=\int_{-\theta / 2}^{\theta / 2} d t\left[\frac{M}{2} \dot{q}^{2}(t)-U(q(t))\right]
$$

is the action of the undamped particle. The imaginary part is evaluated by analytically continuing from real times $t$ to imaginary times $\tau=i t$ (Wick rotation), thereby transforming the complex integrand in $(3.1 \mathrm{~b})$ to a real positive one. Performing the trace in (3.1b) over $q$ yields with $\tau=i \theta$ the partition function $Z$,

$$
Z=\exp (-\beta F)
$$

where the imaginary part of the free energy $F$ is related to the decay rate $\Gamma$ by

$$
\Gamma=\frac{2}{\hbar} \operatorname{Im} F
$$


At temperatures $T>\hbar \omega_{b} / 2 \pi k=T_{0}$, the imaginary part of $F$ is given by the contribution from the saddle point $q(\tau)=q_{b}=$ const. A careful evaluation of the fluctuation modes around the "nucleus" ${ }^{(113)} q(\tau)=q_{b}$ then yields the result ${ }^{(115,116)}$

$$
\operatorname{Im} F \stackrel{T>T_{0}}{\longrightarrow} \frac{k T_{0} \omega_{0}}{2 \omega_{b}}\left(\prod_{n=1}^{\infty} \frac{n^{2} v^{2}+\omega_{0}^{2}}{n^{2} v^{2}-\omega_{b}^{2}}\right) \exp \left(-\beta E_{b}\right)
$$

where $v=2 \pi / \theta$ is the fundamental Matsubara frequency. Observing that the ratio of products in (2.9) is related to elementary functions, one finds for the decay rate $\Gamma$

$$
\Gamma=\frac{\omega_{b}}{2 \pi} \frac{\sin h\left(\frac{1}{2} \theta \omega_{0}\right)}{\sin \left(\frac{1}{2} \theta \omega_{b}\right)} \exp \left(-\beta E_{b}\right), \quad T>T_{0}
$$

In Refs. 95, 96, 98, this result has been obtained by means of a Boltzmann average over energy-dependent transmission coefficients for a parabolic barrier. For $T>T_{0}$, the result in (3.5) is approximated excellently by writing ${ }^{(69)}$

$$
\Gamma \simeq\left[\frac{\omega_{0}}{2 \pi} \exp \left(-\beta E_{b}\right)\right]\left[\exp \frac{\hbar^{2}\left(\omega_{0}^{2}+\omega_{b}^{2}\right)}{24(k T)^{2}}\right], \quad k T>\hbar \omega_{b} / 2 \pi
$$

Note that the result in (3.5) diverges exactly at the crossover temperature $T_{0}$ to quantum tunneling ${ }^{(69,124)}$

$$
T_{0}=\hbar \omega_{b} / 2 \pi k
$$

$T_{0}$ is characterized by the vanishing of the first eigenvalue, $\lambda_{1}=v^{2}-\omega_{b}^{2} \rightarrow 0$ as $T$ approaches $T_{0}$ from above. Alternatively, the periodic bounce trajectory in imaginary time collapses for $T \uparrow T_{0}$ to a constant, $q_{b}$, at precisely the same temperature. Below $T_{0}$, the free energy is dominated by the contribution from the bounce trajectory, $q_{c}(\tau)$, which is a stationary point of the Euclidian (imaginary time) action; i.e., $\delta S_{E}\left(q_{c}\right)=0$, where

$$
S_{E}[q(\tau)]=\int_{-\theta / 2}^{\theta / 2} d \tau\left[\frac{1}{2} M \dot{q}^{2}(\tau)+U(q(\tau))\right]
$$

Thus the solution $q_{c}(\tau)$ obeys a classical equation of motion in the inverted potential $U(q) \rightarrow-U(q)$, i.e.,

$$
M \ddot{q}_{c}=\frac{\partial U}{\partial q_{c}}, \quad q_{c}\left(-\frac{\theta}{2}\right)=q_{c}\left(\frac{\theta}{2}\right)
$$


The period $T\left(E_{\theta}\right) \equiv \theta$

$$
T\left(E_{\theta}\right)=2 M^{1 / 2} \int_{q_{1}(\theta)}^{q_{f}(\theta)}\left\{2\left[U(q)-E_{\theta}\right]\right\}^{-1 / 2} d q \equiv \theta
$$

is equal to the period of a classical orbit in the inverted potential, $-U(q)$, potential- $U(q)$ with energy $-E_{\theta}$. Use of a steepest-descent approximation then gives the result ${ }^{(98)}$

$$
\Gamma=\frac{2 \sinh \left(\frac{1}{2} \beta \hbar \omega_{0}\right)}{\left|2 \pi \hbar T^{\prime}\left(E_{\theta}\right)\right|^{1 / 2}} \exp \left(\frac{-S_{B}}{\hbar}\right), \quad T<T_{0}
$$

where

$$
S_{B}=\int_{-(1 / 2) \theta}^{(1 / 2) \theta} d \tau\left[\frac{M}{2} \dot{q}_{c}^{2}(\tau)+U\left(q_{c}(\tau)\right)\right]
$$

is the Euclidian action evaluated along a single periodic bounce trajectory, and $T^{\prime}$ denotes the derivative $\partial T\left(E_{\theta}\right) / \partial E_{\theta}$.

The behavior close to $T \simeq T_{0}$ is complicated by the nature of the fluctuation modes about the bounce solution $q_{c}(\tau)$ : There is a one-node zero mode (a Goldstone mode; see Fig. 5), proportional to $\dot{q}_{c}(\tau)$; i.e., by virtue of (3.8) we obtain with an additional differentiation

$$
\left[\delta^{2} S_{E}\right] \dot{q}_{c} \equiv\left[-M \frac{d^{2}}{d \tau^{2}}+\left(\frac{d^{2} U}{d q_{c}^{2}}\right)\right] \dot{q}_{c}(\tau)=0
$$

This zero mode describes the phase fluctuations of the bounce solution, and the corresponding nodeless mode has a negative eigenvalue. However, in addition, there is a "dangerous" quasizero mode, ${ }^{(116)}$ describing amplitude fluctuations near $T \simeq T_{0}$. Hence, for $T \simeq T_{0}$, we must go beyond the second variation $\delta^{2} S_{E}$, in the Euclidian action, and take into account the potential shape away from the barrier top. ${ }^{(98.115 .117)}$ This then yields ${ }^{(98)}$

$$
\begin{aligned}
\Gamma= & \frac{\left[2 \sinh \left(\frac{1}{2} \beta \hbar \omega_{0}\right)\right]}{\left|2 \pi \hbar T^{\prime}\left(E_{\theta_{0}}\right)\right|^{1 / 2}} \operatorname{erfc}\left[\left(\beta-\beta_{0}\right)\left|\frac{\hbar}{T^{\prime}\left(E_{\theta_{0}}\right)}\right|^{1 / 2}\right] \\
& \times \exp \left[-\beta E_{b}+\frac{1}{2}\left(\beta-\beta_{0}\right)^{2} \hbar /\left|T^{\prime}\left(E_{\theta_{0}}\right)\right|\right], \quad T \gtrsim T_{0}
\end{aligned}
$$

where $\operatorname{erfc}(x)$ is the function:

$$
\operatorname{erfc}(x)=\frac{1}{\sqrt{2 \pi}} \int_{-\infty}^{x} d t \exp \left(-\frac{1}{2} t^{2}\right)
$$




\subsection{Crossover Temperature}

Next we consider finite quantum damping via a bilinear coupling of the particle to a heat bath with degrees of freedom $\phi_{n}$ (represented by harmonic oscillator normal modes). The system under study is then governed by the Lagrangian

$$
\begin{aligned}
L= & \frac{M}{2} \dot{q}^{2}-U(q)+\sum_{n}\left(\frac{m_{n}}{2} \dot{\phi}_{n}^{2}-\frac{m_{n}}{2} \omega_{n}^{2} \phi_{n}^{2}\right) \\
& -q \sum_{n} \lambda_{n} \phi_{n}-\frac{1}{2} q^{2} \sum_{n} \frac{\lambda_{n}^{2}}{m_{n} \omega_{n}^{2}}
\end{aligned}
$$

Without the last term, which makes the coupling to the heat bath translationally invariant (i.e., it compensates for the coupling-induced renormalization of the potential $\left.{ }^{(109)}\right)$, this model was first studied by Ullersma. $^{(125)}$ It is exactly soluble for a quadratic potential $U(q){ }^{(126,127)}$ Following Ref. 110, one eliminates the degrees of freedom of the bath by tracing out the normal modes $\phi_{n}$. This yields the effective action ${ }^{(109)}$

$$
\begin{aligned}
S[q(t)]= & \int_{-\theta / 2}^{\theta / 2} d t\left[\frac{M}{2} \dot{q}^{2}(t)-U(q(t))\right] \\
& +\frac{1}{2} \int_{-\theta / 2}^{\theta / 2} d t \int_{-\theta / 2}^{\theta / 2} d t^{\prime} K\left(t-t^{\prime}\right)\left[q(t)-q\left(t^{\prime}\right)\right]^{2}
\end{aligned}
$$

The last, nonlocal term is due to the influence of the reservoir. All the properties of the heat bath are contained in the spectral function $J(\omega)$,

$$
J(\omega)=\frac{\pi}{2} \sum_{n} \frac{\lambda_{n}^{2}}{m_{n} \omega_{n}} \delta\left(\omega-\omega_{n}\right)
$$

which in turn determines the function $K(t)$ :

$$
K(t)=\int_{-\infty}^{\infty} \frac{d \omega}{2 \pi} J(\omega)\left\{[1+N(\omega)] e^{i \omega t}+N(\omega) e^{-i \omega t}\right\}
$$

Here, $N(\omega)=1 /(\exp \beta \hbar \omega-1)$ is the Bose-Einstein distribution function. Wick rotating the action as in Section 3.2, observing that $K(t)$ is periodic with period $\theta$ and continuing the imaginary time $\tau$ outside the range $\theta / 2>\tau>-\theta / 2$ by use of the periodic boundary condition $q(\theta+\tau)=q(\tau)$, one obtains for the Euclidian action $S_{E}{ }^{(109,111,115)}$

$$
\begin{aligned}
S_{E}[q(\tau)]= & \int_{-\theta / 2}^{\theta / 2} d \tau\left[\frac{M}{2} \dot{q}^{2}(\tau)+U(q(\tau))\right] \\
& +\frac{1}{2} \int_{-\theta / 2}^{\theta / 2} d \tau \int_{-\infty}^{\infty} d \tau^{\prime} k\left(\tau-\tau^{\prime}\right)\left[q(\tau)-q\left(\tau^{\prime}\right)\right]^{2}
\end{aligned}
$$


where

$$
k(\tau)=\int_{0}^{\infty} \frac{d \omega}{2 \pi} J(\omega) \exp (-\omega|\tau|)
$$

Setting $\delta S_{E}=0$, we obtain the equation obeyed by the dissipation-modified bounce solution ${ }^{(109,115)}$

$$
M \ddot{q}_{c}=\frac{\partial U}{\partial q_{c}}+2 \int_{-\infty}^{\infty} d \tau^{\prime} k\left(\tau-\tau^{\prime}\right)\left[q_{c}(\tau)-q_{c}\left(\tau^{\prime}\right)\right], \quad q_{c}\left(-\frac{\theta}{2}\right)=q_{c}\left(\frac{\theta}{2}\right)
$$

in which the nonlocal part is to be interpreted as its principal value.

A careful study of the bounce equation shows that the highest temperature $T_{0}$, above which the bounce solution $q_{c}(\tau)$ collapses into the constant $q_{b}$, obeys the relation ${ }^{(69)}$

$$
v^{2}-\omega_{b}^{2}+\hat{\gamma}(v) v=0, \quad v=2 \pi / \theta, \quad \theta=\hbar \beta
$$

where $\hat{\gamma}(z)$ is the Laplace transform of the memory damping $\gamma(t)$ [see $(2.55)]$

$$
\hat{\gamma}(z)=\frac{1}{M} \sum_{n} \frac{\lambda_{n}^{2} z}{m_{n} \omega_{n}^{2}\left(z^{2}+\omega_{n}^{2}\right)}
$$

The same relation (3.20) was encountered previously, (2.59), in the study of the escape rate in systems with frequency-dependent friction. Therefore, in terms of the memory-renormalized reactive frequency $\tilde{\mu}$ in $(2.59)$, we have for the crossover temperature $T_{0}$, below which tunneling events predominate over thermally activated Arrhenius-type transitions, the result $^{(69)}$

$$
T_{0}=\hbar \tilde{\mu} / 2 \pi k \simeq\left(1.2157 \times 10^{-12} \sec \mathrm{K}\right) \tilde{\mu}
$$

To put it differently, the ratio $\tilde{\mu} / \omega_{b}$, which gives the difference between the simple transition-state rate, (2.1), (2.2), and the Kramers rate, (2.58), also determines the deviation of the simple undamped crossover temperature (3.7) from the correct, dissipation, and memory-renormalized result (3.22). The behavior of the crossover temperature $T_{0}$ is depicted in Fig. 6 as a function of the dimensionless damping strength $\kappa$ and the memory correlation time $\tau_{c}$ for two different memory kernels $\gamma(t)$ : (a) $\gamma(t)=\omega_{b} \kappa J_{1}\left(\kappa \omega_{b} t / r\right) / t$, where $J_{1}$ is the Bessel function of first kind, and (b) an exponential memory $\gamma(t)=\left(\kappa \omega_{b} / \tau_{c}\right) \exp \left(-t / \tau_{c}\right) ; \gamma_{0}=\kappa \omega_{b}$. 

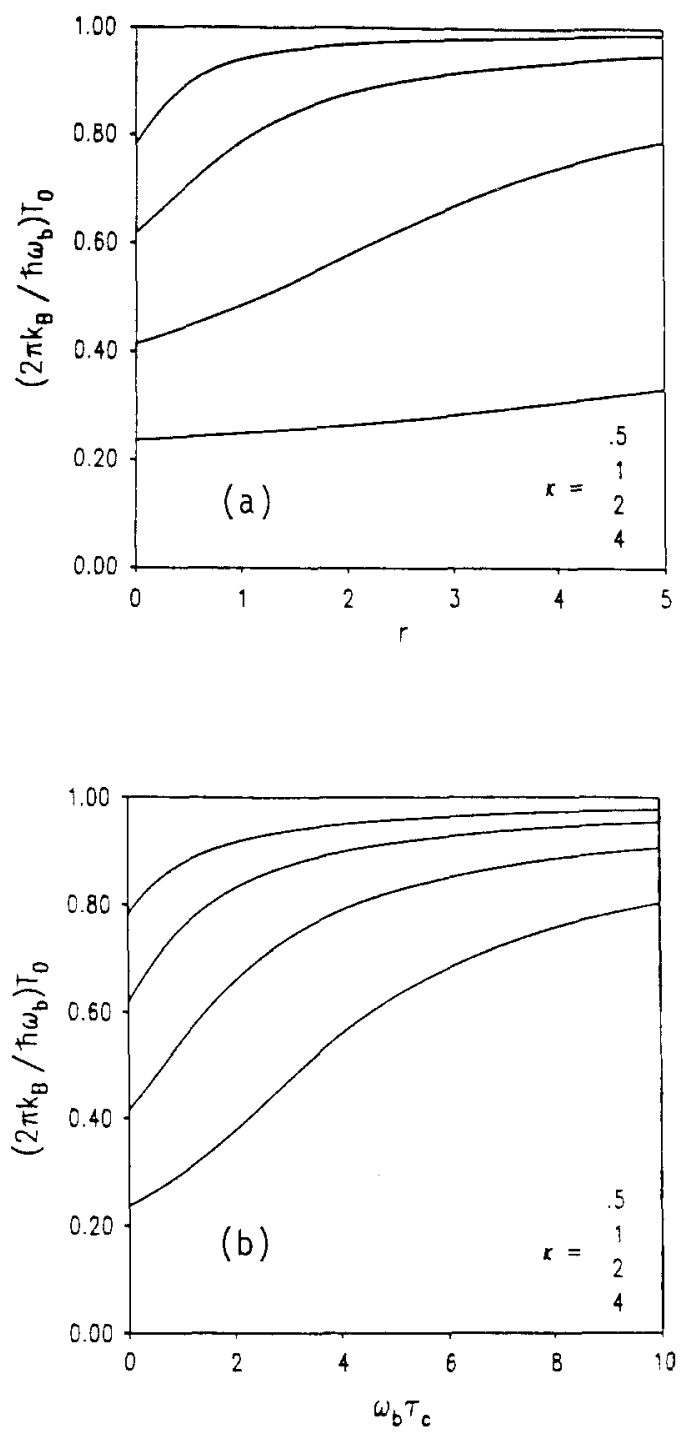

Fig. 6. (a) The dimensionless crossover temperature $\left(2 \pi k / \hbar \omega_{b}\right) T_{0}=\tilde{\mu} / \omega_{b}$ for model (a) is depicted versus the memory-correlation time parameter $r$ for different values of the dimensionless noise strength $\kappa=\gamma_{0} / \omega_{b}$. (b) Same as Fig. 6a for an exponential memory (model b) with noise strength $\gamma_{0}=\kappa \omega_{b}$ and dimensionless memory correlation time $\omega_{b} \tau_{c}$. 
A determination of this crossover temperature $T_{0},(3.22)$, which for weak dissipation is readily observable on an Arrhenius plot of the rate $^{(128,129)}$, gives valuable information about both the form of the barrier and the dissipation mechanism.

\subsection{Quantum Version of the Kramers Approach}

We are now in a position to consider the quantum theory of the Kramers rate in (2.20). In order for the Ehrenfest equation for the tunnelling coordinate, $q$, to be of the form in (2.6) (ohmic damping), we must use a spectral density $J(\omega),(3.16)$, given by ${ }^{(109)}$

$$
J(\omega)=M \gamma \omega
$$

With this choice one finds

$$
k(\tau)=\frac{M \gamma}{2 \pi} \frac{1}{\tau^{2}}
$$

Upon a partial integration of (3.19), the bounce equation for ohmic damping reads explicitly $(\varepsilon>0)$

$$
\begin{aligned}
-\ddot{q}_{c}+\frac{1}{M} \frac{\partial U\left(q_{c}\right)}{\partial q_{c}}=\frac{\gamma}{2 \pi} \int_{-\infty}^{\infty} d \tau^{\prime} \frac{\partial q_{c}}{\partial \tau^{\prime}} \times\left(\frac{1}{\tau^{\prime}-\tau+i \varepsilon}+\frac{1}{\tau^{\prime}-\tau-i \varepsilon}\right) & \\
q_{c}\left(-\frac{\theta}{2}\right) & =q_{c}\left(\frac{\theta}{2}\right)
\end{aligned}
$$

At finite temperatures $T<T_{0}$, this equation can be solved exactly for a cubic potential in the limit of very weak, very strong and at one particular moderate friction value $\gamma^{(115)}$

Let us first evalute the quantum Kramers rate for temperatures above $T_{0}$. In this case, the Gaussian fluctuation modes around the minimum $q_{0}$ [we normalize the potential $U(q)$ so that $U\left(q_{0}\right)=0$ ] are seen to possess the eigenvalues $(v=2 \pi / \theta)$

$$
\lambda_{n}^{0}=n^{2} v^{2}+\omega_{0}^{2}+|n| \gamma v, \quad n=0, \pm 1, \pm 2, \ldots
$$

and the eigenvalues of modes around the constant bounce $q_{c}(\tau)=q_{b}$ are obtained as ${ }^{9}$

$$
\lambda_{n}^{b}=n^{2} v^{2}-\omega_{b}^{2}+|n| \gamma v, \quad n=0, \pm 1, \pm 2 \ldots
$$

\footnotetext{
${ }^{9}$ With a memory damping, $\hat{\gamma}(z)$, one only needs to substitute in $(3.26)$ and $(3.27): \gamma \rightarrow \hat{\gamma}(|n| v)$.
} 
With these sets of eigenvalues, the result for $\operatorname{Im} F$ in (3.4) is generalized to give for the rate (116,130,131) $^{(1)}$

$$
\begin{aligned}
\Gamma & =\frac{2}{h} \operatorname{Im} F=\left[\frac{\Gamma\left(1-\lambda^{+} / v\right) \Gamma\left(1-\lambda^{-} / v\right)}{\Gamma\left(1-\eta^{+} / v\right) \Gamma\left(1-\eta^{-} / v\right)}\right] \frac{\mu}{\omega_{b}} \frac{\omega_{0}}{2 \pi} \exp \left(-\beta E_{b}\right) \\
& =\rho \Gamma^{\text {Kramers }}, \quad T>T_{0}
\end{aligned}
$$

where

$$
\lambda^{ \pm}=\frac{-\gamma}{2} \pm\left(\frac{\gamma^{2}}{4}+\omega_{b}^{2}\right)^{1 / 2}, \quad \eta^{ \pm}=\frac{-\gamma}{2} \pm\left(\frac{\gamma^{2}}{4}-\omega_{0}^{2}\right)^{1 / 2}
$$

This result was obtained first by Wolynes in Ref. 130. Here, we made use of the fact that the corresponding ratio of products [see (3.4)] can be expressed in terms of $\Gamma$ functions. The quantity $\rho$ in $(3.28 \mathrm{~b})$ measures the deviation from the classical rate (2.20) and approaches unity for $T \gg T_{0}$. More importantly, the result in (3.28) diverges exactly at the crossover temperature $T_{0}$, where a more careful treatment is needed. ${ }^{(115-117)}$ Interestingly enough, an accurate working approximation for the quantity $\rho$, which is valid independent of the dissipative mechanism (i.e., ohmic or non-ohmic) reads ${ }^{(69)}$ (see Fig. 7)

$$
\rho \simeq \exp \left[\frac{\hbar^{2}}{24} \frac{\left(\omega_{0}^{2}+\omega_{b}^{2}\right)}{(k T)^{2}}+O\left(T^{-4}\right)\right]
$$

This quantum correction only renormalizes the Arrhenius factor but does not depend on dissipation - in contrast to $T_{0}$, being proportional to $\tilde{\mu}$; see (3.22) and (2.59).

For temperatures $T \leqslant T_{0}$, the evaluation of $\operatorname{Im} F$ is more delicate. Following the reasoning of Riseborough, Hanggi, and Freidkin (Ref. 115), we present here the treatment of the quantum fluctuations below $T \lesssim T_{0}$. The escape rate, evaluated in the approximation of a dilute gas of bounces, is given by the ratio of contributions to the Green's function (3.1) from the paths close to the bounce trajectory $q_{c}(\tau)$ relative to the paths which always remain close to the metastable minimum at $q_{0}$. Since these contributions are dominated by the extrema of the Euclidian action, the rate is controlled by the exponential of the bounce action relative to the action of the path $q(\tau)=q_{0}, U\left(q_{0}\right)=0$. The exponential part

$$
\Gamma \propto \exp \left[-S_{B}(\theta, \gamma) / \hbar\right]
$$

given by the dissipation-renormalized single bounce action at temperature $T$ matches smoothly, at the crossover temperature $T_{0}$, with the Arrhenius 


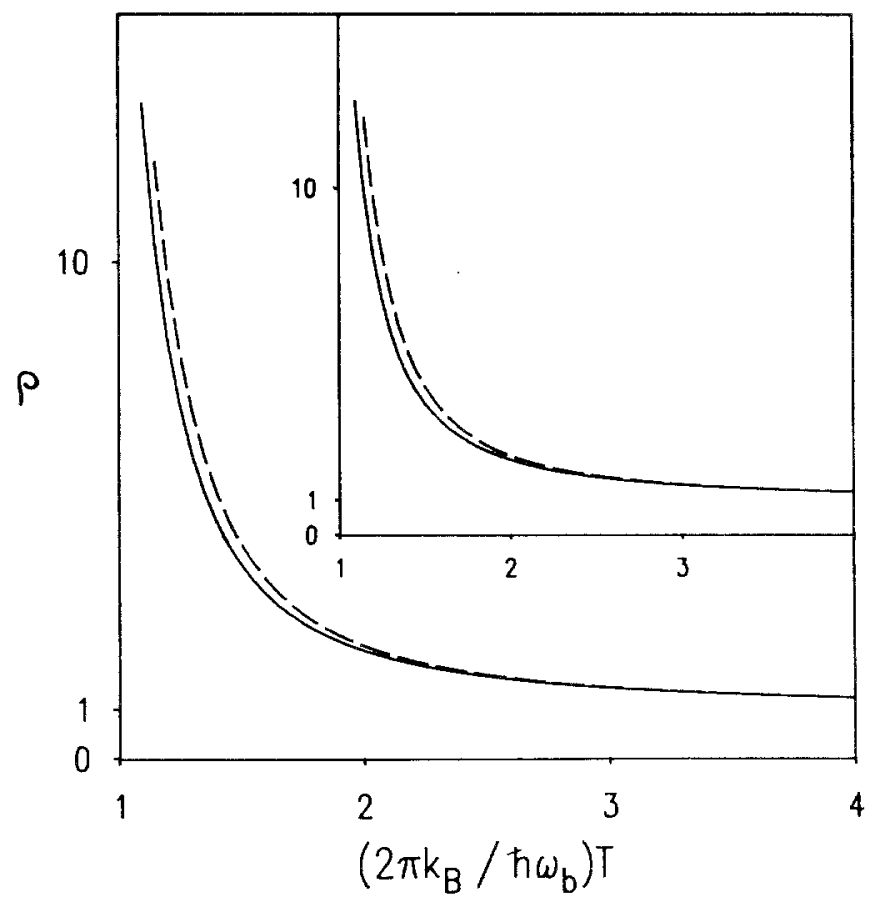

Fig. 7. The approximation (3.29) (dashed line), for the quantum correction factor $\rho$ is compared with the theoretical result (3.26)-(3.28) (solid line), for model (a) with parameter values $\omega_{0}=\omega_{b}, \kappa=0.5$, and $r=0.5$. The inset shows the same for model (b) with parameter values $\omega_{0}=\omega_{b}, \kappa=0.5, \omega_{b} \tau_{c}=0.5$ (taken from Ref. 69).

factor $S_{B}\left(\theta_{0}, \gamma\right) / \hbar=E_{b} / k T_{0}{ }^{(111)}$ The prefactor can be written as the ratio of the small fluctuations about these extremal paths. If one sets for the partion function $Z$, (3.2), $Z=Z_{0}-i Z_{B} \times \exp \left(-S_{B} / \hbar\right)$, the decay rate $\Gamma$ is simply

$$
\Gamma=\frac{2}{\hbar} \operatorname{Im} F=\frac{2 Z_{B}}{\left(\theta Z_{0}\right)} \exp \left(-S_{B} / h\right)
$$

For $T<T_{0}$, this can be shown to reduce to ${ }^{(111,115-118)}$

$$
\begin{gathered}
\Gamma=\left\{\frac{1}{2 \pi \hbar} \int_{-\theta / 2}^{\theta / 2} d \tau\left[\dot{q}_{c}(\tau)\right]^{2}\right\}^{1 / 2}\left(\frac{\operatorname{Det}\left(\delta^{2} S_{E} / \delta q^{2}\right)_{q=q_{0}}}{\left|\operatorname{Det}^{\prime}\left(\delta^{2} S_{E} / \delta q^{2}\right)_{q=q_{c}}\right|}\right)^{1 / 2} \\
\times \exp \left[-S_{B}(\theta, \gamma) / \hbar\right], \quad T<T_{0}
\end{gathered}
$$

where Det' means that the eigenvalue zero has to be omitted. For a detailed derivation of this result we refer the reader to Ref. 115, wherein we provide additional insight and give further details on the formalism. In par- 
ticular, near $T \simeq T_{0}$, the evaluation of $Z_{B}$ must be modified slightly, owing to the occurrence of two zero modes. Apart from the Goldstone mode $\left(\sim \dot{q}_{c}\right)$, this new zero mode at $T=T_{0}$ corresponds again to amplitude fluctuations about $q_{c}(\tau){ }^{(116)}$ Both zero modes can be eliminated by considering the effect that the cubic and quartic interactions, $\delta^{3} S_{E}$ and $\delta^{4} S_{E}$, have. ${ }^{(115-117)}$ Moreover, near $T \simeq T_{0}$ there exists a frequency scale $\Gamma_{0}$ and a temperature scale $x_{0}$ (those depend on the particular system under consideration) such that in the regime $\left|T-T_{0}\right| \lesssim x_{0}$, the rate $\Gamma$ exhibits a universal scaling behavior; i.e. $\Gamma / \Gamma_{0}=\operatorname{Erfc}(\chi) \exp \left(\chi^{2}\right), \chi=\left(T-T_{0}\right) / x_{0} \cdot{ }^{(116)}$

Below $T_{0}$, the role of the Arrhenius factor is taken over by the dissipation-modified single bounce action $S_{B}(\theta, \gamma) \equiv S_{E}\left[q_{c}\right]$, (3.18a), which grows continuously to its zero temperature value $S_{B}(\theta=\infty, \gamma)$. Near $T \simeq 0$, the rate $\Gamma$ exhibits a universal temperature enhancement given by ${ }^{(111)}$

$$
S_{B}(T, \gamma)=S_{B}(T=0, \gamma)-\left(M \omega_{0}\left[\bar{q}-q_{0}\right]^{2}\right) \frac{\pi \gamma}{6 \omega_{0}}\left(k T \tau_{B} / h\right)^{2}
$$

wherein $\tau_{B}$ is the zero temperature bounce length: i.e., with $U\left(q_{0}\right)=U(\bar{q})=0, \bar{q}>q_{0}$

$$
\tau_{B}=\frac{1}{\left(\bar{q}-q_{0}\right)} \int_{-\infty}^{\infty} q_{c}(T=0, \gamma ; \tau) d \tau
$$

This power law enhancement, $\left(\ln \Gamma \propto T^{2}\right)$, of the rate is independent of the particular form of the metastable potential $U(q)$ and is valid for any ohmiclike dissipative mechanism obeying $\hat{\gamma}(z=0)=\gamma_{0}>0 .{ }^{(11)}$ As a matter of fact, recent experiments ${ }^{(132,133)}$ have verified many of these specific lowtemperature features of ohmiclike quantum tunneling. In particular, the unique ohmic behavior $\ln [\Gamma(T) / \Gamma(T=0)] \propto T^{2}(T \rightarrow 0)$ has been observed experimentally first in Ref. 132a together with the characteristic decrease of $\Gamma(T, \gamma)$ with increasing dissipation strength. ${ }^{(111,115)}$ An analytical treatment of the prefactor in (3.32) is possible, but not simple. ${ }^{(115)}$ In practice, one must therefore resort to numerical methods. ${ }^{(134,135)}$ In particular, one finds that the prefactor in (3.32) is only very weakly dependent on temperature. $^{(135,136)}$ For $T \simeq 0$, the prefactor $A(T, \gamma)$ actually obeys the same power law, ${ }^{(136)} \ln A \sim-c(\gamma) T^{2}+O\left(T^{4}\right), c(\gamma)>0$; however, this decrease of the prefactor is far too weak to offset the increase in the exponential factor given by the bounce action $S_{B}(\theta, \gamma) .^{(111)}$

\section{ACKNOWLEDGMENTS}

The author would like to thank Hermann Grabert, Gert Ingold, Peter Riseborough, and Ulrich Weiss for an enjoyable collaboration in tunneling problems. Also, many stimulating critical comments by these collaborators, as well as those by my colleagues Michal Borkovec, Peter Talkner, and 
Andy Zangwill, have added greatly to the form and content of this paper. Moreover, the author has benefited from helpful discussions with Markus Buttiker, Benny Carmeli, David Chandler, Sudip Chakravarty, James Hynes, Rolf Landauer, Anthony Leggett, Bernie Matkowsky, Frank Moss, and Attila Szabo. This work was supported in part by the Joint Service Electronics Program and ONR grant N00014-85-K-0372.

\section{REFERENCES}

1. H. A. Kramers, Physica (Utrecht) 7:284 (1940).

2. S. Chandrasekhar, Rev. Mod. Phys. 15:1, 63-68 (1943).

3. H. C. Brinkman, Physica (Utrecht) 22:149 (1956).

4. R. Landauer and J. A. Swanson, Phys. Rev. 121:1668 (1961).

5. J. S. Langer, Phys. Rev. Lett. $21: 973$ (1968); Ann. Phys. (N.Y.) 54:258 (1969).

6.(a) B. Widom, J. Chem. Phys. 55:44 (1971); (b) H. Tomita, A. Ito, and H. Kidachi, Progr. Theor. Phys. 56:786 (1976); (c) P. B. Visscher, Phys. Rev. B 13:3272 (1976); 14:347 (1976); (d) C. Blomberg, Physica (Utrecht) 86A:49 (1977); (e) N. G. Van Kampen, Suppl. Progr. Theor. Phys. 64:389 (1978); (f) R. S. Larson and M. D. Kostin, J. Chem. Phys. 72:1392 (1980); (g) J. L. Skinner and P. G. Wolynes, J. Chem. Phys. 69:2143 (1978); 72:4913 (1980); (h) J. R. Montgomery, D. Chandler, and B. J. Berne, J. Chem. Phys. 70:4065 (1979); B. J. Berne, J. L. Skinner, and P. G. Wolynes, J. Chem. Phys. 73:4314 (1980); (i) R. F. Grote and J. T. Hynes, J. Chem. Phys. 74:4465 (1981); (j) M. Buttiker, E. P. Harris, and R. Landauer, Phys. Rev. B 28:1268 (1983); (k) M. Buttiker and R. Landauer, Phys. Rev. Lett. 52:1250 (1984).

7. H. Frauenfelder and P. G. Wolynes, Science 229:337 (1985).

8. J. Kurkijarvi, Phys. Rev. B 6, 832 (1972); L. D. Jackel, W. W. Webb, J. E. Lukens, and S. S. Pei, Phys. Rev. B 9:115(1974).

9. T. A. Fulton and L. N. Dunkleberger, Phys. Rev. B 9:4760 (1974).

10. W. de Boer and R. de Bryn Ouboter, Physica (Utrecht) 98B:185 (1980); Physica (Utrecht) 122B:1 (1983).

11. E. G. D. D'Agliano, P. Kumar, W. Schaich, and H. Suhl, Phys. Rev. B 11:2122 (1975); Proc. 24th Nobel Symposium on Collective Properties of Physical Systems (Academic Press, New York, 1973).

12. G. Iche and Ph. Nozières J. Phys. (Paris) 37:1313 (1976); B. A. Huberman and J. B. Boyce, Solid State Commun. 25:843 (1978).

13. R. D. Young, J. Chem. Phys. 80:554 (1984).

14. D. Beece, L. Eisenstein, H. Frauenfelder, D. Good, M. C. Marden, L. Reinisch, A. H. Reynolds, L. B. Sorensen and K. T. Yue, Biochemistry 19:5147 (1980).

15. W. Doster, Biophysical Chemistry 17:97 (1983).

16. S. Mashimo, Macromolecules 9:91 (1976).

17. D. L. Hasha, T. Eguchi, and J. Jonas, J. Chem. Phys. 75:1570 (1981); J. Am. Chem. Soc. 104:2290 (1982).

18. G. Rothenberger, D. K. Negus, and R. M. Hochstrasser, J. Chem. Phys. 79:5360 (1983).

19. S. P. Velsko, D. H. Waldeck, and G. R. Fleming, J. Chem. Phys. 78:249 (1983); J. Chem. Phys. 65:59 (1982); S. H. Courtney and G. R. Fleming, Chem. Phys. Lett. 103:443 (1984); B. Bagchi and D. Oxtoby, J. Chem. Phys. 78:2735 (1983).

20. B. Otto, J. Schroeder, and J. Troe, J. Chem. Phys. 81:202 (1984); H. Hippler, V. 
Schubert, and J. Troe, J. Chem. Phys. 81:3931 (1984); H. Hippler and J. Troe, Int. J. Chem. Kin. 8:501 (1976).

21. J. C. Tully, Surf. Sci. 111:461 (1981); C. Caroli, B. Roulet and D. Saint-James, Phys. Rev. $B$ 18:545 (1978).

22. S. Glasstone, K. J. Laidler, and H. Eyring, The Theory of Rate Processes (McGraw-Hill, New York, 1941); H. Eyring, J. Chem. Phys. 3:107, 492 (1935).

23. C. A. Wert and C. Zener, Phys. Rev. 76:1169 (1949); H. Fröhlich, The Theory of Dielectric (Calendron Press, Oxford, 1950).

24. A. Seeger, Encyclopedia of Physics, Vol. VII, part 1 (Springer, Berlin, 1955).

25. G. H. Vineyard, J. Phys. Chem. Solids 3:121 (1957).

26. S. A. Rice, Phys. Rev. 112:804 (1958); S. A. Rice and H. L. Frisch, J. Chem. Phys. 32:1046 (1960).

27. N. B. Slater, Theory of Unimolecular Reactions (Methuen, London, 1959); J. Chem. Phys. 24:1256 (1956).

28. H. R. Glyde, Rev. Mod. Phys. 39:373 (1967); K. J. Laidler and A. Tweedale, Adv. Chem. Phys. 21:113 (1971), in particular see p. 117.

29. P. Pechukas, Ann. Rev. Phys. Chem. 29:59 (1978); Ber. Bunsenges. Phys. Chem. 86:372 (1982).

30. C. W. Gardiner, J. Stat. Phys. 30:157 (1983).

31. G. H. Vineyard and J. Krumhansl, Phys. Rev. B 31:4929 (1985).

32. H. Grabert, P. Hanggi, and P. Talkner, J. Stat. Phys. 22:537 (1980).

33. K. Kawasaki, J. Phys. A6:1289 (1973).

34. O. Klein, Ark. Mat. Astr. Fys. 16, No 5 (1922).

35. H. D. Vollmer and H. Risken, Physica (Utrecht) 110A:106 (1982); K. Voigtlaender and H. Risken, Chem. Phys. Lett. 105:506 (1984); J. Stat. Phys. 40:397 (1985).

36. H. Risken and K. Voigtlaender, J. Stat. Phys. 41:825 (1985).

37. G. Wilemski, J. Stat. Phys. 14:153 (1976); U. M. Titulaer, Physica (Utrecht) 91A:321 (1978); 100A:251 (1980); H. Risken, H. D. Vollmer, and M. Morsch, Z. Phys. B40:343 (1980); P. Grigolini and F. Marchesoni, in Memory function approaches to stochastic problems in condensed matter, Adv. Chem. Phys. 62, 1, 29 (1985); Physica (Utrecht) 121A:269 (1983); M. San Miguel and J. M. Sancho, J. Stat. Phys. 22:605 (1980); F. Haake, Z. Phys. B48:31 (1982).

38. M. V. Smoluchowski, Ann. Phys. (Leipzig) 21:756 (1906); 48:1103 (1915); Phys. Z. 17:557, 585 (1917).

39. P. Hanggi and H. Thomas, Phys. Rep. 88C:207-319 (1982), Section 3.2.

40. P. Hanggi, Helv. Phys. Acta 51:202 (1978); Z. Naturforsch. 33A:1380 (1978); P. Hanggi, F. Rosel, and D. Trautmann, Z. Naturforch. 33A:402 (1978); G. Grosso and G. Pastori Parravicini, Adv. Chem. Phys. 62:81 (1985).

41. H. Risken, The Fokker-Planck Equation (Springer Series in Synergetics, No. 18, Springer, New York, 1984).

42. U. Weiss, Phys. Rev. A 25:2444 (1982) (Rapid Commun.); U. Weiss and W. Haffner, in Functional Integration, J. P. Antoine and E. Tirapegui, eds. (Plenum, New York, 1980).

43. G. H. Weiss, Adv. Chem. Phys. 13:1 (1969).

44. B. J. Matkowsky and Z. Schuss, SIAM J. Appl. Math. 33:365 (1977); Z. Schuss, SIAM Rev. 22:119 (1980); see also D. Ludwig, SIAM Rev. 17:605 (1975).

45. P. Hanggi and P. Talkner, Phys. Rev. Lett. 51:2242 (1983); Z. Phys. B45:79 (1981); Phys. Rev. A 32:1934 (1985); P. Talkner and P. Hanggi, Phys. Rev. A 29:768 (1984); P. Talkner and D. Ryter, in Noise in Physical Systems and l/f Noise, M. Savelli, G. Lecay, and J. P. Nougier, eds. (Elsevier Science Publ., New York, 1983).

46. S. R. Shenoy and G. S. Agarwal, Phys. Rev. A 29:1315 (1984); S. R. Shenoy, Phys. Rev. A 30:2849 (1984) (Rapid Commun.) 
47. K. Schulten, Z. Schulten, and A. Szabo, J. Chem. Phys. 74:4426 (1981); see also A. Szabo, K. Schulten, and Z. Schulten, J. Chem. Phys. 72:4350 (1980).

48. G. H. Weiss and A. Szabo, Physica (Utrecht) 119A:569 (1983).

49. M. V. Smoluchowski, Z. Phys. Chem. 92:129 (1917).

50. P. Debye, Trans. Electro-Chem. Soc. 82:265 (1942).

51. F. C. Collins and G. E. Kimball, J. Colloid Sci. 4:425 (1949).

52. G. Wilemski and M. Fixman, J. Chem. Phys. 58:4009 (1972).

53. D. Shoup and A. Szabo, Biophys. J. 40:33 (1982).

54. F. A. Lindemann, Trans. Faraday Soc. 17:589 (1922); E. W. Montroll and K. E. Shuler, Adv. Chem. Phys. 1:361 (1958); J. Troe, Ann. Rev. Chem. Phys. 29:223 (1978); J. Chem. Phys. 66:4745 (1977); T. A. Bak and J. L. Lebowitz, Phys. Rev. 131:1138 (1963); S. E. Nielsen and T. A. Bak, J. Chem. Phys. 41:665 (1964).

55. M. Buttiker and R. Landauer, Phys. Rev. B 30:1551 (1984).

56. P. Hanggi and U. Weiss, Phys. Rev. A 29:2265 (1984).

57. M. Buttiker, in 17th Int. Conf. Low Temp. Physics, Karlruhe 1984, U. Eckern et al., eds. (Elsevier Science Publ., Amsterdam, 1984), EP 18, p. 1155.

58. S. H. Northrup and J. A. Mc Cammon, J. Chem. Phys. 72:4569 (1980); G. Van der Zwan and J. T. Hynes, J. Chem. Phys. 77:1295 (1982); S. H. Northrup and J. A. McCammon, $J$. Chem. Phys. 78:987 (1983).

59. M. Borkovec and B. J. Berne, J. Chem. Phys. 82:794 (1985).

60. B. J. Matkowsky, Z. Schuss, and E. Ben-Jacob, SIAMJ. Appl. Phys. 42:835 (1982).

61. P. Hanggi and F. Mojtabai, Phys. Rev. A 26:1168 (1982) (Rapid Commun.); P. Hanggi, Springer Proc. Phys. 1:95 (1984).

62. S. A. Adelman, J. Chem. Phys. 64:124 (1976).

63. R. F. Fox, Phys. Rep. 48C:179 (1978).

64. P. Hanggi and H. Thomas, Z. Phys. B26:85 (1977); H. Grabert, P. Hanggi, and P. Talkner, Z. Phys. B26:389 (1977); P. Hanggi, H. Thomas, H. Grabert, and P. Talkner, $J$. Stat. Phys. 18:155 (1978).

65. R. F. Grote and J. T. Hynes, J. Chem. Phys. 73:2715 (1980); J. T. Hynes, in The Theory of Chemical Reaction Rates, M. Baer, ed. (CRC Press, Boca Raton, Florida, in press).

66. P. Hanggi, J. Stat. Phys. 30:401 (1983).

67. B. Carmeli and A. Nitzan, Phys. Rev. A 29:1481 (1984).

68. M. M. Dygas, B. J. Matkowsky, and Z. Schuss, SIAM Appl. Math. (in press).

69. P. Hanggi, H. Grabert, G. Ingold, and U. Weiss, Quantum theory of activated events in presence of long time memory, Phys. Rev. Lett. 55:761 (1985).

70. J. E. Straub, M. Borkovec, and B. J. Berne, J. Chem. Phys. 83:3172 (1985); J. Chem. Phys. (in press).

71. R. W. Zwanzig, Phys. Fluids 2:12 (1959).

72. B. Carmeli and A. Nitzan, Phys. Rev. Lett. 49:423 (1982); R. F. Grote and J. T. Hynes, J. Chem. Phys. 77:3736 (1982); A. Nitzan, J. Chem. Phys. 82:1614 (1985); A. G. Zawadzki and J. T. Hynes, Chem. Phys. Lett. 113:476 (1985).

73. B. J. Matkowsky, Z. Schuss, and C. Tier, J. Stat. Phys. 35:443 (1984).

74. G. Nicolis, Rep. Progr. Phys. 42:225 (1979).

75. N. G. Van Kampen, Stochastic Processes in Physics and Chemistry (North-Holland, Amsterdam, 1981).

76. W. Horsthemke and R. Lefever, Noise Induced Transitions, Theory and Applications in Physics, Chemistry and Biology (Series in Synergetics, Vol. 15, Springer, New York, 1984); Fluctuations and Sensitivity in Nonequilibrium Systems (Springer Proc. Phys. Vol. I, eds. W. Horsthemke and D. K. Kondepudi, Springer, New York, 1984).

77. R. L. Stratonovich, Topics in the Theory of Random Noise, Vols. I and II (Gordon and Breach, New York, 1963/1966). 
78. R. Landauer, J. Appl. Phys. 33:2209 (1962); Phys. Today 31:23 (1978).

79. V. De Giorgio and M. O. Scully, Phys. Rev. A 2:1170 (1970); R. Graham and H. Haken, Z. Phys. 237:31 (1970); R. Graham, Springer Tracts in Modern Physics 66:1 (1973); H. Haken, Rev. Mod. Phys. 47:67 (1975); J. F. Scott, M. Sargent III, and C. Cantrell, Opt. Commun. 15:13 (1975).

80. E. Abraham and S. D. Smith, Rep. Progr. Phys. 45:815 (1982); L. A. Lugiato, Progr. Optics 21:69 (1984); R. Bonifacia, M. Gronchi, and L. A. Lugiato, Phys. Rev. A18:2266 (1978); P. Hanggi, A. R. Bulsara, and R. Janda, Phys. Rev. A 22:671 (1980).

81. P. Hanggi, Phys. Lett. 78A:304 (1978); S. Faetti, P. Grigolini, and F. Marchesoni, Z. Phys. B47:353 (1982).

82. M. H. Devoret, J. M. Martinis, D. Esteve, and J. Clarke, Phys. Rev. Lett. 53:1260 (1984); T. Fonesca and P. Grigolini, Phys. Rev. A (in press).

83. P. Hanggi and P. Riseborough, Am. J. Phys. 51:347 (1983).

84. L. N. Epele, H. Fanchiatti, M. Spina, and H. Vucetich, Phys. Rev. A 31:2631 (1985).

85. M. Buttiker and R. Landauer, in Nonlinear Phenomena at Phase Transitions and Instabilities, T. Riste, ed. (Plenum, New York, 1982), pp. 111-143.

86. V. Ambegaokar and B. J. Halperin, Phys. Rev. Lett. 22:1364 (1969); Yu. M. Ivanchenko and L. A. Zilberman, Sov. Phys. JETP 28:1272 (1969).

87. E. Ben-Jacob, D. J. Bergman, B. J. Matkowsky, and Z. Schuss, Phys. Rev. A 26:2805 (1982); E. Ben-Jacob, D. J. Bergman, Y. Imry, B. J. Matkowsky, and Z. Schuss, J. Appl. Phys. 54:6533 (1983); H. D. Vollmer and H. Risken, Z. Phys. 52B:259 (1983); P. Jung and H. Risken, Z. Phys. B54:357 (1984).

88. P. Fulde, L. Pietronero, W. R. Schneider, and S. Strassler, Phys. Rev. Lett. 35:1776 (1975); T. Geisel, Physics in Superionic Conductors, M. B. Salamon, ed. (Springer, New York 1979), p. 201; G. Gruner, A. Zawadovski, and P. M. Chaikin, Phys. Rev. Lett. 46:511 (1981).

89. P. Hanggi and H. Thomas, Phys. Rep. 88C:207 (1982), Section 4.3.

90. R. Graham and T. Tél, J. Stat. Phys. $35: 729$ (1984); 37:709 (1984) (Addendum); Phys. Rev. A 31:1109 (1985); 31:3364 (1985).

91. P. Hanggi, F. Marchesoni, and P. Grigolini, Z. Phys. B56:333 (1984); P. Hanggi, T. J. Mroczkowski, F. Moss, and P.V.E. McClintock, Phys. Rev. A 32: 695 (1985) (Rapid Commun.).

92. P. Hanggi and P. Riseborough, Phys. Rev. A 27:3379 (1983) (Rapid Commun.); C. Van den Broeck and P. Hanggi, Phys. Rev. A 30:2730 (1984).

93. P. Hanggi, H. Grabert, P. Talkner, and H. Thomas, Phys. Rev. A 29:371 (1984).

94. B. J. Matkowsky, Z. Schuss, C. Knessl, C. Tier, and M. Mangel, Phys. Rev. A 29:3359 (1984).

95. E. Wigner, Z. Phys. Chem. B19:203 (1932); Trans. Faraday Soc. $24: 29$ (1938).

96. R. P. Bell, Proc. R. Soc. (London) 13:204 (1933); Trans. Faraday Soc. 55:1 (1959); R. P. Bell, The Tunnel Effect in Chemistry (Chapman and Hall, London, 1980).

97. G. Alefeld, Phys. Rev. Lett. 12:372 (1964); C. P. Flynn, Phys. Rev. 171:682 (1982).

98. I. Affleck, Phys. Rev. Lett. 46:388 (1981); see also U. Weiss and W. Haeffner, Phys. Rev. D 27:2916 (1983).

99. R. Pirc and P. Gosar, Phys. Kond. Mater. 9:377 (1969).

100. L. M. Sander and H. B. Shore, Phys. Rev. B 3:1472 (1969); B12:1546 (1975).

101. J. A. Sussmann, Ann. Phys. (Paris) 6:135 (1971).

102. T. Holstein, Ann. Phys. (N.Y.) 8:325 (1959); 8:343 (1959); D. Emin and T. Holstein, Ann. Phys. (N.Y.) 53:439 (1969).

103. C. P. Flynn and A. M. Stoneham, Phys. Rev. 1B:3966 (1970).

104. J. J. Hopfield, Proc. Natl. Acad. Sci. 71:3640 (1974); in Tunneling in Biological SSystems, B. Chance et al., eds. (Academic Press, New York, 1979). 
105. W. A. Phillips, Philos. Mag. 34:983 (1976).

106. P. Riseborough, Phys. Stat. Solidi 117B:381 (1983); Ann. Phys. (N.Y.) 153:1 (1984).

107. J. P. Sethna, Phys. Rev. B24:698 (1981); B25:5050 (1982); doctoral thesis, Princeton University, 1981.

108. A. J. Leggett, J. Phys. (Paris) C6:1264 (1978); Progr. Theor. Phys. Suppl, 69:80 (1980); 80:10 (1984).

109. A. O. Caldeira and A. J. Leggett, Phys. Rev. Lett. 46:211 (1981); Ann. Phys. (N.Y.) 149:374 (1983); 153:445 (1984) (Erratum).

110. R. P. Feynman and A. R. Hibbs, Quantum Mechanics and Path Integrals (McGraw-Hill, New York, 1965); R. P. Feynman, Statistical Mechanics (Benjamin, New York, 1972), Chap. 3.

111. H. Grabert, U. Weiss and P. Hanggi, Phys. Rev. Lett. 52:2193 (1984); see also Refs. 69, 115, 116 and H. Grabert, in SQUID85, H. D. Hahlbohm and H. Lubbig (eds.), (deGruyter, Berlin 1985).

112. R. P. Feynman, Phys. Rev. 97:660 (1955); R. P. Feynman and F. L. Vernon, Ann. Phys. (N.Y.) 24, 118 (1963); A. O. Caldeira and A. J. Leggett, Physica (Utrecht) 121A:587 (1983); A. Schmid, J. Low Temp. Phys. 49:609 (1982).

113. J. S. Langer, Ann. Phys. (N.Y.) 41:108 (1967).

114. S. Coleman, Phys. Rev. D 15:2929 (1977); C. G. Callan and S. Coleman, Phys. Rev. D 16:1762 (1977); S. Coleman, in The Whys of Subnuclear Physics, A. Zichichi, ed. (Plenum, New York, 1979).

115. P. Riseborough, P. Hanggi, and E. Freidkin, Phys. Rev. A 32:489 (1985).

116. H. Grabert and U. Weiss, Phys. Rev. Lett. 53:1787 (1984).

117. A. I. Larkin and Yu. N. Ovchinnikov, Sov. Phys. JETP 59:420 (1984).

118. A. Schmid, Quasiclassical wave function in multidimensional quantum decay problems, preprint, 1985.

119. T. Banks, C. M. Bender, and T. T. Wu, Phys. Rev. D 8:3346 (1973); 8:3366 (1973); H. J. de Vega, J. L. Gervais, and B. Sakita, Phys. Rev. D 19:604 (1979).

120. U. Weiss. P. Riseborough, P. Hanggi, and H. Grabert, Phys. Lett. 104A:10 (1984); 104A:492 (1984) (Erratum)

121. S. Chakravarty and A. J. Leggett, Phys. Rev. Lett. 52:5 (1984); S. Chakravarty, Phys. Rev. Lett. 49:681 (1982); A. J. Bray and M. A. Moore, Phys. Rev. Lett. 49:1546 (1982); B. Carmeli and D. Chandler, J. Chem. Phys. 82:3400 (1985).

122. S. Chakravarty and S. Kivelson, Phys. Rev. Lett. 50:1811 (1983); 51:1109 (1983) (Erratum); Phys. Rev. B 32:76 (1985); H. Grabert and U. Weiss, Phys. Rev. Lett. 54:1605 (1985); M. P. A. Fisher and A. Dorsey, Phys. Rev. Lett. 54:1609 (1985).

123. P. Hanggi and $P$. Riseborough, unpublished; approximate weak friction quantum results, valid for $T>\hbar \omega_{b} / 2 \pi k$, can be found in V. I. Melnikov, Sov. Phys. JETP 60:380 (1984).

124. V. I. Goldanskii, Dokl. Akad. Nauk. SSSR 124:1261 (1959); 127:1037 (1959).

125. P. Ullersma, Physica (Utrecht) 32:27, 56, 74, 90 (1966).

126. R. J. Rubin, Phys. Rev. 131:964 (1963).

127. H. Grabert, U. Weiss, and P. Talkner, Z. Phys. B55:87 (1984); P. Riseborough, P. Hanggi, and U. Weiss, Phys. Rev. A 31:471 (1985); F. Haake and R. Reibold, Acta Physica Austriaca 56:37 (1985); Phys. Rev. A 32: 2462 (1985).

128. H. Frauenfelder, in Tunneling in Biological Systems, B. Chance et al. eds. p. $627 \mathrm{ff}$, (Academic Press, New York, 1979); p. 627 ff N. Alberding, R. H. Austin, K. W. Beeson, S. S. Chan, L. Eisenstein, H. Frauenfelder, and T. M. Nordlund, Science 192:1002 (1976).

129. R. Di Foggio and R. Gomer, Phys. Rev. B 25:3490 (1982); W. Rieheman and E. Nembach, J. Appl. Phys. 55:1081 (1984).

130. P. G. Wolynes, Phys. Rev. Lett. 47:968 (1981).

131. V. I. Melnikov and S. V. Meshkov, JETP Lett. 38:130 (1983). 
132. S. Washburn, R. A. Webb, R. F. Voss, and S. M. Faris, Phys. Rev. Lett. 54: 2712 (1985); R. F. Voss and R. A. Webb, Phys. Rev. Lett. 47:265 (1981); L. D. Jackel et al. Phys. Rev. Lett. 47:697 (1981); R. J. Prance et al., Nature 289:543 (1981).

133. D. B. Schwartz, B. Sen, C. N. Archie, A. K. Jain, and J. E. Lukens, Phys. Rev. Lett. 55:1547 (1985).

134. L. D. Chang and S. Chakravarty, Phys. Rev. B 29:130 (1984); 30:1566 (1984) (Erratum).

135. H. Grabert, P. Olschowski, and U. Weiss, Phys. Rev. B 32:3348 (1985) (Rapid Commun.); Phys. Rev. Lett. (Comment) (in press).

136. P. Riseborough, J. Stat. Phys. (in press). 


\section{Addendum and Erratum:}

\section{Escape from a Metastable State [J. Stat. Phys. 42:105-148 (1986)] Peter Hanggi}

In this note I update the above article with some relevant material and correct some minor misprints. Following eq. (2.7) one should state more precisely: If $U(x)$ is a nonlinear and nonquadratic potential field, analytical exact results for (2.7) within the full damping regime are not known. For a parabolic potential field (linear force), $U(x)=(M / 2) \omega_{0}^{2} x^{2}$, the eigenvalues and eigenfunctions are readily obtained (Ref. 41 ). In particular, the complex-valued eigenvalues read

$$
\lambda_{n, m}=-\frac{1}{2}(n+m) \gamma+\frac{1}{2} i\left[4 \omega_{0}^{2}-\gamma^{2}\right]^{1 / 2}(n-m) \quad n, m=0,1, \ldots
$$

Approximate, analytical solutions for a bistable potential at intermediate and high friction can be found in: J. F. Gouyet and A. Bunde, J. Stat. Phys. 36:43 (1984); Physica 132A:357 (1985).

In the case of the strongly overdamped (Smoluchowski) case the boundary condition following (2.24) should be corrected to read

$$
p_{o}\left(x \sim x_{1}\right)=\bar{p}\left(x \sim x_{1}\right)
$$

For weakly damped systems, the equilibrium probability $\bar{p}(E),(2.41)$, for the energy depends, of course, on the inverse density of states $v(E)$ on the dimension of the reaction coordinate $\mathbf{x}$. Equation (2.53c) should be completed by writing for the density of states $\psi(E)$

$$
\psi(E)=\int d \Omega \delta[E-\mathscr{H}(\mathbf{\Omega})] \equiv 1 / \nu(E)
$$

The multidimensional generalization of (eqs. 2.38, 2.41-2.46, 2.51, 2.52, and 2.62 is then given with $v(E)$ determined from $(2.53 \mathrm{c})$. Moreover, it should also be noted that the rate exhibits in the weak friction regime a rather 


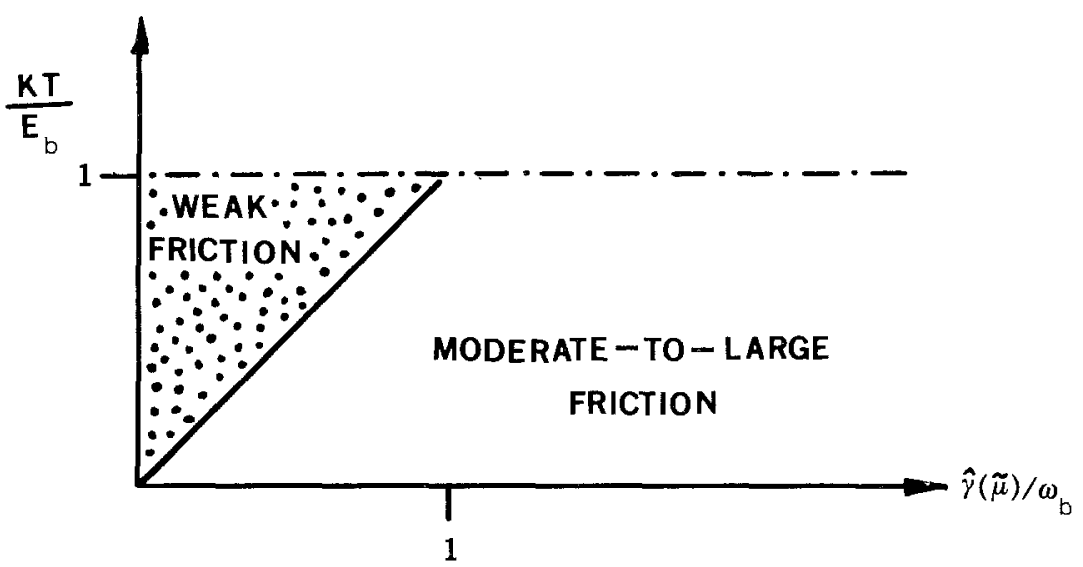

Fig. 1. Regime of validity of the various classical rate expressions as a function of the dimensionless parameters $k T / E_{b}$ and $\hat{\gamma}(\tilde{\mu}) / \omega_{b}$, with $\tilde{\mu}$ (memory friction) defined in (2.59). Weak-friction results (energy diffusion controlled regime): eqs. 2.43, 2.44, 2.45, 2.46, and 2.48; moderate-to-large friction: eqs. $2.20,2.49$, and 2.58; crossover regime: eqs. 2.46 and 2.64 .

interesting sensitivity to the coupling strengths among the degrees of freedom $\mathbf{x}$, modeling the reaction dynamics. Recently, this has been beautifully demonstrated in a study by M. Borkovec, J. E. Straub, and B. J. Berne, "The Influence of Intramolecular Vibrational Relaxation on the Pressure Dependence of Unimolecular Rate Constants", J. Chem. Phys. 85:(in press).

Reference $70 \mathrm{~b}$, containing the informative numerical simulation data for escape driven by exponentially correlated Gaussian noise, should be completed as follows: J. E. Straub, M. Borkovec, and B. J. Berne, J. Chem. Phys. 84:1788 (1986). Also, Ref. 109 should be complemented by adding the reference: A. J. Leggett, Phys. Rev. B, 30:1208 (1984). In Ref. 20 we should add: G. Maneke, J. Schroeder, J. Troe, and F. Voss, Ber. Bunsenges. Phys. Chem. 89:896 (1985).

Finally, we present here a rate-phase diagram which summarizes the regime of validity of the various classical rate expressions (Fig. 1).

\section{ACKNOWLEDGMENT}

Research supported by the Joint Service Electronics Program, contract Nos. F49620-85-C-0078 and F49620-82-C-0084. 